\title{
A FUNDAMENTAL IMPROVEMENT TO ERICKSEN-LESLIE KINEMATICS
}

\author{
BY
}

HOSSEIN POURMATIN (Civil and Environmental Engineering, Carnegie Mellon University),

AMIT ACHARYA (Civil and Environmental Engineering, Carnegie Mellon University),

AND

KAUSHIK DAYAL (Civil and Environmental Engineering, Carnegie Mellon University)

\begin{abstract}
We demonstrate theory and computations for finite-energy line defect solutions in an improvement of Ericksen-Leslie liquid crystal theory. Planar director fields are considered in two and three space dimensions, and we demonstrate straight as well as loop disclination solutions. The possibility of static balance of forces in the presence of a disclination and in the absence of flow and body forces is discussed. The work exploits an implicit conceptual connection between the Weingarten-Volterra characterization of possible jumps in certain potential fields and the Stokes-Helmholtz resolution of vector fields. The theoretical basis of our work is compared and contrasted with the theory of Volterra disclinations in elasticity. Physical reasoning precluding a gauge-invariant structure for the model is also presented.
\end{abstract}

1. Introduction. While liquid crystal theory, especially that of nematics, is by now a well-established branch of condensed matter physics [KL03, there is one aspect in which the classical theory may be considered deficient. Disclination line defects are an integral part of the physics of liquid crystals; however, there does not exist a classical theory, whether Oseen-Frank for statics or Ericksen-Leslie in dynamics (which naturally subsumes the static theory), that predicts bounded energy in a finite body containing a $\pm \frac{1}{2}$-strength disclination line. It is perhaps for this reason that it is commonplace amongst workers in liquid crystal theory to associate a line defect only with a singularity; e.g. the 'escape' solution for a +1 strength line defect [CK72] is often not referred to as a defect solution.

Director fields with infinite energy are practically problematic from the point of view of finding solutions; e.g. in statics, energy cannot be minimized in a class of functions

Received May 25, 2013.

2010 Mathematics Subject Classification. Primary 76A15.

E-mail address: mpourmat@andrew.cmu.edu

E-mail address: acharyaamit@cmu.edu

E-mail address: kaushik@cmu.edu 
where all competing fields with defects have undefined (infinite) energies. Practical difficulties remain in dynamics, more so if the goal is to specify dynamics of defect lines. While for the purpose of static Oseen-Frank theory involving a few defects one overcomes such problems by excluding from analysis a core region of small volume along the defect line where one assumes the theory does not hold and ascribing a finite energy to it from some unspecified-in-theory nonlinear effects, for situations where large numbers of defects may be involved, such an approach is untenable. To quote Ericksen [Eri91]: "I am interested in seeing the development of a mathematically sound theory of defects which might be at rest, or moving....serious difficulties are encountered with some observed kinds of disclinations (line defects). According to the aforementioned theories [OseenFrank, Ericksen-Leslie], these more violent singularities cause energy integrals, etc., to diverge. In dealing with rather specific situations, workers have patched up the theory by excluding a tube of small radius, assigning a finite 'core energy' to it. I just do not believe that one can use such ideas as a basis for developing satisfactory mathematical theory, particularly for moving defects. Such phenomena are of interest for both nematic and cholesteric liquid crystals. In the latter, they are associated with the interesting 'blue phases'...."

In AD12 a theory for the dynamics of non-singular line defects has been proposed as an extension of Ericksen-Leslie Les92 theory. In a sense, it is inspired by the goal of exploring the deeper connections between the classical theory of defects, originating in elasticity theory from the works of Weingarten and Volterra, and the theory of liquid crystals, as begun in the work of Kléman Klé73. The model works with augmented kinematics involving the director field and an incompatible director distortion field as a replacement for the director gradient field. The main point of departure is that the director distortion is not curl-free in general. The curl of the field has the kinematic meaning of an areal density of lines carrying a vectorial attribute and is referred to as the director incompatibility field.

For the 1-constant Oseen-Frank energy approximation, it was shown in AD12 that the developed formalism can indeed predict the outside-core, energy density distribution of Frank's planar director distributions for line defects with strength given by all integer multiples of $\frac{1}{2}$. Moreover, all such distributions have finite energy in the whole body (including core). The core is represented by precisely the region where the director incompatibility field is non-vanishing. However, it was not shown in that work how to extract a physically realistic continuous director field resembling Frank's planar defect fields on the whole body, and neither was it shown what form the director distortion tensor might take within the theory to represent planar distortion fields of disclination line defects. In this paper we achieve that goal.

The modeling of bounded energy line defects in statics started with the pioneering work of CK72 within classical Oseen-Frank theory; an interesting perspective on boundedenergy line defects is provided in [Set85. The same question served as motivation for the development of the variable degree of orientation extension of classical Ericksen-Leslie theory in [Eri91. Within the De-Gennes $Q$ tensor formalism it has been pursued in the pioneering work of Schopohl and Sluckin [SS87, followed by SKH95, KVicv99, [BZ07], MZ10], to provide a representative sample. In the dynamic case, representative 
studies are those of [HKGLGCC08] and YFMW09] within the $Q$-tensor approach and [BS05, BS12] and [LL00,LW00] within the Ericksen-Leslie approach; in particular, BS12. provides a noteworthy balanced perspective on the philosophy of modeling liquid crystal defects by the $Q$-tensor approach or the director-field-singularity approach. In LL00, LW00 non-singular line-defect solutions appear due to relaxation of the unit-magnitude constraint on the director field. In contrast, in our work the demonstrated finite energy defect solutions satisfy the strict unit-vector constraint on the director field.

The defect solutions we develop here are finite energy analogs of classically accepted 'energy-minimizing' (infinite total energy) singular solutions of Oseen-Zocher-Frank theory. As is customary in the liquid crystal literature when discussing statics of defects solutions, we (almost) ignore balance of forces. What we are able to show in this regard (Section 5) is that static balance of linear momentum is satisfied outside the core(s) of disclination(s) in the absence of a body force within our model (assuming an incompressible fluid). Moreover, when there is a single disclination in the body, the natural boundary conditions for static force and moment balance representing no applied forces and couples on the boundary imply that the resultant force on any surface enveloping the disclination core vanishes. Within the core, we discuss possibilities afforded by the AD12 theory in satisfying balance of linear momentum without external body forces or flow.

We consider this work to be primarily a characterization of the kinematic augmentation of the Ericksen-Leslie theory achieved by the AD12 model, since results are not presented regarding the connection of the presented solutions to (quasi)equilibria (cf. [CP89]) of the latter.

This paper is organized as follows: in Section 2 we provide notation. Section 3 describes the analytical basis of our work; Section 4 describes the finite-element based numerical scheme employed to compute non-singular defect solutions and the results. In Section 5 we discuss static balance of forces for the developed solutions, and Section 6 ends with some concluding remarks. Two appendices provide explicit solutions that are necessary for discussing our results.

2. Notation. A "." represents the inner product of two vectors, while ":" represents the trace inner product of two second-order tensors, $\boldsymbol{A}: \boldsymbol{B}=A_{i j} B_{i j}$.

In rectangular Cartesian coordinates and corresponding bases and components, for a vector field $\boldsymbol{A}$ and a scalar field $\theta$ we write

$$
\begin{array}{r}
\operatorname{div} \boldsymbol{A}=A_{i, i}, \\
(\operatorname{curl} \boldsymbol{A})_{i}=e_{i j k} A_{k, j}, \\
(\operatorname{grad} \theta)_{i}=\theta_{, i} .
\end{array}
$$

For a second-order tensor field $\boldsymbol{A}$, we define

$$
(\operatorname{curl} \boldsymbol{A})_{i m}=e_{m j k} A_{i k, j} \text {. }
$$

The following list describes some of the mathematical symbols we use in this paper:

$\boldsymbol{n}$ : director

$\boldsymbol{E}$ : director distortion tensor 
$\boldsymbol{E}^{\theta}$ : director distortion vector

$\boldsymbol{\beta}$ : director incompatibility tensor

$\boldsymbol{\beta}^{\theta}$ : director incompatibility vector

$K$ : defect strength

$\nu$ : normal vector

$\boldsymbol{T}$ : Cauchy stress tensor

$\Lambda$ : couple stress tensor

$\psi$ : free energy per unit mass

$p:$ pressure

$\theta$ : angle of the director field

$\lambda$ : layer field

$\boldsymbol{\lambda}_{\perp}$ : incompatible part of $\boldsymbol{\lambda}$

$\operatorname{grad} z$ : compatible part of $\boldsymbol{\lambda}$

3. Theory. We consider the static (i.e. director and positional inertia-less) governing equations of the framework introduced in $\mathrm{AD} 12$ and demonstrate that there exist finiteenergy states in the model that are solutions corresponding to line defects with planar director distributions within the Oseen-Frank constitutive assumption.

3.1. Governing equations. Static Balance of Angular Momentum is given by the statement

$$
\Lambda_{i j, j}-e_{i j k} T_{j k}=0 .
$$

Ericksen's identity for the theory (i.e. a necessary condition for frame-indifference of the energy function) is the statement

$$
e_{i j k}\left(\frac{\partial \psi}{\partial n_{i}} n_{j}+\frac{\partial \psi}{\partial E_{i r}} E_{j r}-E_{r i} \frac{\partial \psi}{\partial E_{r j}}\right)=0 .
$$

Static Balance of Linear Momentum is the statement

$$
T_{i j, j}=0 .
$$

For a free-energy density of the form $\psi(\boldsymbol{n}, \boldsymbol{E})$, the constitutive statements for the stress and couple stress tensors for the theory AD12 read as

$$
\begin{aligned}
\Lambda_{i j} & =e_{i r s} n_{r} \frac{\partial \psi}{\partial E_{s j}}, \\
T_{i j} & =-p \delta_{i j}-E_{r i} \frac{\partial \psi}{\partial E_{r j}},
\end{aligned}
$$

where $p$ is the pressure arising from the constraint of incompressibility. Using these statements along with the Ericksen identity in angular momentum balance with some manipulations yields the fundamental governing partial differential equation of our work:

$$
e_{i r s}\left[n_{s} \frac{\partial \psi}{\partial n_{r}}-\left(E_{r j}-n_{r, j}\right) \frac{\partial \psi}{\partial E_{s j}}+n_{r}\left(\frac{\partial \psi}{\partial E_{s j}}\right)_{, j}\right]=0 .
$$

Note the interesting fact that when $E_{r j}=n_{r, j}$, i.e. there is no director incompatibility and hence no defects, we have the classical statement of angular momentum balance in statics Ste04]. Inasmuch, this term in our theory may be interpreted as giving one 
explicit form to Ericksen's "internal body moments" [Eri91, $\boldsymbol{g}^{I}$, arising from the presence of defects in the body.

We record the statement of balance of linear momentum,

$$
-p_{, i}-\left(E_{r i} \frac{\partial \psi}{\partial E_{r j}}\right)_{, j}=0,
$$

to be used later in Section 5 .

For the sake of this paper, we consider an ansatz consisting of director distributions parametrizable by a single angle field $\theta$ on the body and director distortion fields of the form

$$
\boldsymbol{E}:=\frac{\partial \boldsymbol{n}}{\partial \theta} \otimes \boldsymbol{E}^{\theta}
$$

where $\boldsymbol{E}^{\theta}$ is a vector field with possibly non-vanishing curl. When there are no line defects, we require $\boldsymbol{E}^{\theta}=\operatorname{grad} \theta$ so that $\boldsymbol{E}=\operatorname{grad} \boldsymbol{n}$.

Let the core region for a given $\boldsymbol{E}^{\theta}$ field on the body be the set of points on which

$$
\boldsymbol{\beta}^{\theta}:=\operatorname{curl} \boldsymbol{E}^{\theta} \neq \mathbf{0}
$$

and

$$
\boldsymbol{\beta}:=\operatorname{curl}(\boldsymbol{E}-\operatorname{grad} \boldsymbol{n}) \neq \mathbf{0} .
$$

We refer to an $\boldsymbol{E}^{\theta}$ field as containing an isolated line defect if its $\boldsymbol{\beta}^{\theta}$ field is non-zero only in a cylindrical region that forms a closed loop or extends from one boundary of the body to another.

The goal now is to construct pairs of $(\boldsymbol{E}, \boldsymbol{n})$ fields

(1) that satisfy (11);

(2) that produce Frank's isolated line defect-like energy density fields outside the core region while producing bounded energy in finite bodies (including the core region) when no moments are applied on the boundary of the body;

(3) that produce Frank's wedge disclination-like director distributions except the core and possibly another region of 'small', but non-zero, volume in the body;

(4) for which the line integral of $\boldsymbol{E}^{\theta}$ along any closed contour surrounding the core of an isolated line defect of strength $K$ evaluates to $2 \pi K$.

A generic result of our paper is as follows: Up to particular boundary conditions for specific problems, pairs of fields $(\boldsymbol{E}, \boldsymbol{n})$ that satisfy the above four requirements for the 1-constant Oseen-Frank energy given by (5) can be generated from (3), (4), (7), (13), and (18). In the following section we provide the rationale behind such a result.

3.2. Construction of bounded energy distortion and director solutions. We work with a rectangular Cartesian coordinate system with unit vectors $\boldsymbol{e}_{i}, i=1,2,3$. $\theta$ represents the angle of the director measured counter-clockwise from the $x_{1}$-axis in the $x_{1}-x_{2}$ plane (looking down the $x_{3}$-axis). Thus

$$
\boldsymbol{n}=\cos \theta \boldsymbol{e}_{1}+\sin \theta \boldsymbol{e}_{2} .
$$

For the sake of illustrating the essentials of our approach, we work with the 1-constant Oseen-Frank energy density approximation

$$
\psi(\boldsymbol{n}, \boldsymbol{E})=\frac{1}{2} \kappa \boldsymbol{E}: \boldsymbol{E}=\frac{1}{2} \kappa E_{i j} E_{i j}=\frac{1}{2} \kappa E_{i}^{\theta} E_{i}^{\theta}
$$


and

Noting that

$$
\frac{\partial \psi}{\partial E_{s j}}=\kappa E_{s j}=\kappa \frac{\partial n_{s}}{\partial \theta} E_{j}^{\theta}
$$

and (5)-(6), (11) reduces to

$$
E_{r j}-n_{r, j}=\frac{\partial n_{r}}{\partial \theta}\left(E_{j}^{\theta}-\theta_{, j}\right)
$$

$$
e_{i r s} n_{r} \kappa\left[\frac{\partial n_{s}}{\partial \theta} E_{j}^{\theta}\right]_{, j}=0 .
$$

Considering (44) and realizing that $e_{i r s} n_{r} \frac{\partial^{2} n_{s}}{\partial \theta^{2}} \theta_{, j}=0$, static angular momentum balance reduces to

which is satisfied if and only if

$$
e_{i r s} n_{r} \frac{\partial n_{s}}{\partial \theta} E_{j, j}^{\theta}=0
$$

$$
\operatorname{div} \boldsymbol{E}^{\theta}=0 .
$$

To fulfill the fourth requirement on the field $\boldsymbol{E}^{\theta}$, let us now consider a distribution of $\boldsymbol{\beta}^{\theta}:=\operatorname{curl} \boldsymbol{E}^{\theta}$ in a right-cylinder parallel to $x_{3}$ such that

$$
\int_{A} \boldsymbol{\beta}^{\theta} \cdot \boldsymbol{e}_{3} d x_{1} d x_{2}=2 \pi K
$$

where $A$ is the set of points representing the cross-section of the core cylinder. Thus, in addition to (6), we require

$$
\operatorname{curl} \boldsymbol{E}^{\theta}=\boldsymbol{\beta}^{\theta} .
$$

Finally, to impose the fact that we seek non-trivial solutions for an unloaded body, we impose vanishing moments on the boundary of the body which, under the current ansatz, are satisfied if

$$
\boldsymbol{E}^{\theta} \cdot \boldsymbol{\nu}=0
$$

on the boundary of the body with normal field $\nu$.

It is straightforward to see that for a specified field $\boldsymbol{\beta}^{\theta}$ on a simply-connected domain, (17)-(9)-(10) have a unique solution. In [AD12, these equations are solved explicitly for a radially symmetric $\boldsymbol{\beta}^{\theta}$ distribution (see Appendix $(\mathrm{B})$ ), and the outside-core result is shown to be exactly the same as the gradient of the Frank angle field for straight wedge disclinations. In the general case (i.e. not necessarily radially-symmetric $\boldsymbol{\beta}^{\theta}$ field, but still localized in core cylinder distributions), we would now like to extract the outsidecore result into a gradient of a continuous everywhere scalar angle field $\theta$ which will serve to define the $\boldsymbol{n}$ field of the pair $(\boldsymbol{E}, \boldsymbol{n})$ describing an isolated defect. Of course, in the process we would like to have $\boldsymbol{E}^{\theta}$ still be the solution to (7)-(9)-(10) and $\boldsymbol{E}^{\theta}=\operatorname{grad} \theta$ when $\boldsymbol{\beta}^{\theta}=\mathbf{0}$.

To perform this extraction, suppose for a moment it is possible to construct a squareintegrable vector field $\boldsymbol{\lambda}$ such that

$$
\operatorname{curl} \boldsymbol{\lambda}=-\boldsymbol{\beta}^{\theta}
$$

on the body, where the $\boldsymbol{\beta}^{\theta}$ field is identical to the one occurring in (9). Then a StokesHelmholtz resolution of the field $\boldsymbol{\lambda}$ can be written as

$$
\boldsymbol{\lambda}=\boldsymbol{\lambda}_{\perp}+\operatorname{grad} z
$$




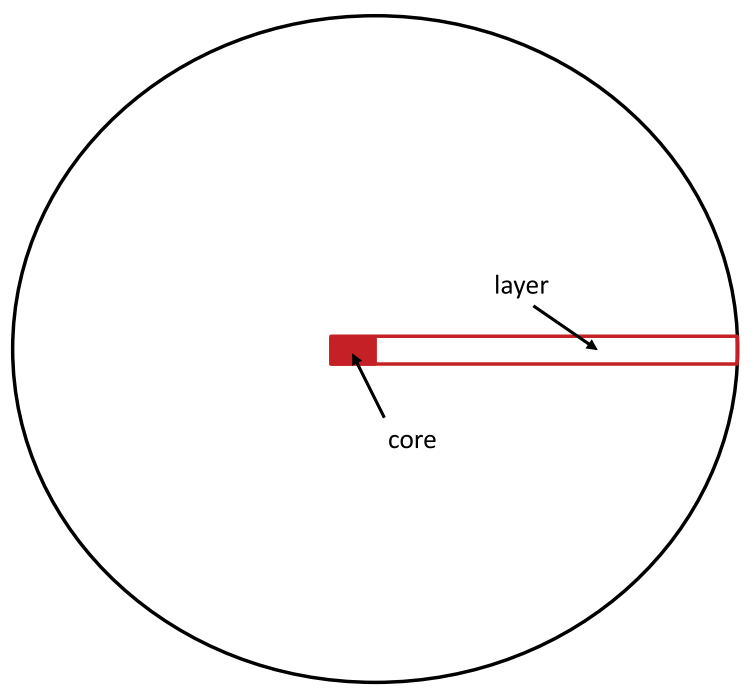

FIG. 1. Schematic of cross-section of a 3-d body showing layer and core geometry.

with

$$
\begin{gathered}
\operatorname{curl} \boldsymbol{\lambda}_{\perp}=\operatorname{curl} \boldsymbol{\lambda}=-\boldsymbol{\beta}^{\theta} \\
\operatorname{div} \boldsymbol{\lambda}_{\perp}=0
\end{gathered}
$$

and

$$
\boldsymbol{\lambda}_{\perp} \cdot \boldsymbol{\nu}=0 \text { on boundary of body, }
$$

and because of the uniqueness of solutions to (7)-(9)-(10), $\boldsymbol{E}^{\theta}=-\boldsymbol{\lambda}_{\perp}$.

Suppose further now that the field $\boldsymbol{\lambda}$ is non-vanishing only on a layer-like region of non-zero volume 1 that may be visualized as a terminating fattened 2 -d surface which, moreover, contains as a proper subset the core cylinder on which $\boldsymbol{\beta}^{\theta}$ has support (Figure 11).

Then, as an immediate consequence we have that

$$
-\operatorname{grad} z=\boldsymbol{\lambda}_{\perp}=-\boldsymbol{E}^{\theta} \quad \text { outside the layer. }
$$

Thus, if we were to now declare the potential $z$ as the required angle-field $\theta$, we would have completed the needed extraction.

In practice (Section 4), we implement the above idea by defining

$$
\boldsymbol{E}^{\theta}:=\operatorname{grad} \theta-\boldsymbol{\lambda}
$$

and requiring this combination to satisfy (77)-(9)-(10) so that

$$
\operatorname{grad} \theta-\boldsymbol{\lambda}=-\boldsymbol{\lambda}_{\perp}
$$

is enforced by uniqueness implying $\operatorname{grad} \theta=\operatorname{grad} z$. When accompanied by the requirement that $\boldsymbol{\lambda}=\mathbf{0}$ if $\boldsymbol{\beta}^{\theta}=\mathbf{0}$, the strategy ensures that $\boldsymbol{E}=\operatorname{grad} \boldsymbol{n}$ in the absence of

\footnotetext{
${ }^{1}$ Cf. DeW73] DeWit considers a distributional layer field in the context of disclinations in solids related to defects in positional order.
} 
defects in the body. We note the important fact that the Stokes-Helmholtz decomposition of an $L_{2}$ vector field implies that $\operatorname{grad} z$ is square-integrable and therefore $\operatorname{grad} \theta$ cannot have a non-square-integrable singularity in the body. The set of definitions (3) and (13) constitutes a primary result of this paper.

So, the only question that remains is whether such a layer-field $\boldsymbol{\lambda}$ satisfying (11) can in fact be constructed. As we show in Section 4, it suffices to consider the layer region as in Figure 1, prescribe an appropriate constant vector field (representative of an interpolation of the required jump in the value of $\theta$, i.e. $2 \pi K$, in the direction transverse to the layer) for the values of $\boldsymbol{\lambda}$ outside the core in it, and taper this interpolated jump to zero over the width of the core. Outside the layer, $\boldsymbol{\lambda}$ is assigned to vanish. Since $\boldsymbol{\lambda}$ represents a transverse 'gradient' in the layer whereas in the core this transverse variation has a gradient in an in-plane direction, it cannot have vanishing curl in the core. Thus we represent a non-singular, but localized, core of a line defect.

We now ask the question of the region where $\boldsymbol{\beta}$ is non-vanishing corresponding to an isolated wedge disclination. The existence of a differentiable extracted $\theta$ field (which can be arranged as can be seen from the construction in Appendix (A) implies that the $\boldsymbol{\beta}$ field corresponding to an isolated defect would be non-vanishing at most in the layer, since $\operatorname{grad} \theta=\boldsymbol{E}^{\theta}$ outside the layer and

$$
E_{r j, k}=\frac{\partial^{2} n_{r}}{\partial \theta^{2}} \theta_{, k} E_{j}^{\theta}+\frac{\partial n_{r}}{\partial \theta} E_{j, k}^{\theta} .
$$

Our use of a layer field may be interpreted as a regularized analog of the possible terminating discontinuity in the displacement field over a 'cut' surface in the WeingartenVolterra (WV) process of elastic dislocation theory, adapted to a much simpler situation than elasticity; thus, the construction shows direct links between the Stokes-Helmholtz (SH) resolution of a vector field (in this instance, the layer field) and the WeingartenVolterra process, in particular, how the 'curl' part of SH encodes, through a smooth field, topological content that can only be represented by a nasty singularity in the gradient of a discontinuous potential field in the WV process. Also, how the 'gradient' part of $\mathrm{SH}$ of an appropriately designed vector field (the layer field) can represent most of the characteristics of the discontinuous potential field of the WV process away from the discontinuity.

3.3. Does topological defect density determine the director distribution? The construction above lays bare an interesting fact. Note that $\boldsymbol{E}^{\theta}$ is uniquely determined by the defect density field $\boldsymbol{\beta}^{\theta}$ (and balance of moments) but $\operatorname{grad} \theta$, and hence the predicted director distribution, is defined by the compatible part $\operatorname{grad} z$ of $\boldsymbol{\lambda}$. An alternative way of seeing this is to substitute

$$
\boldsymbol{E}^{\theta}=\operatorname{grad} \theta-\boldsymbol{\lambda}_{\perp}-\operatorname{grad} z
$$

into (7)-(9)-(10) (this idea is not restricted to the 1-constant energy in any way). Thus, while the energy density distribution, and therefore the couple stress, can be correctly predicted purely from the knowledge of the defect distribution (in this static setting), uniquely predicting the director distribution requires additional physical input in the presence of defects. In particular, the layer-like $\boldsymbol{\lambda}$ field consistent with a specified isolated defect density field orients the non-singular director distribution of the defect, as is shown 
in Section 4. To draw an association with classical potential theory, specifying the surface of discontinuity of a scalar potential field whose gradient is required to match, except on the discontinuity surface, a prescribed, smooth, irrotational vector field on a doublyconnected region containing a toroidal or through-hole eliminates the vast non-uniqueness associated with such definition of a potential otherwise. The argument also reveals why the classical theory with only the director field as a degree of freedom is necessarily limited in the context of modeling defects.

It is also interesting to observe that this situation is entirely analogous to the extension that was required for making a prediction of permanent deformation [Ach01] in the elastic theory of continuously distributed dislocations [Krö81, Wil67]. Comparing and contrasting with prevalent notions in gauge theories, $\theta$ and the total displacement field of plasticity theory are not inconsequential fields to be gauge-transformed away even when they have no energetic cost through the Lagrangian in the presence of gauge fields $(\boldsymbol{\lambda}$, in this context), but direct physical observables that participate in understanding energy dissipation, shape changes, and optical response of the body. Hence, their evolution requires additional physical specification beyond the topological defect density field. Gauge theoretic perspectives on the modeling of nematic defects may be found in Sar82. and [KB85].

3.4. Relationship with disclinations in elasticity theory. Our construction of the $\boldsymbol{\lambda}$ field draws direct motivation from a standard question of potential theory related to understanding the allowable jumps of a scalar potential across a 2-d surface in a multiplyconnected domain described by a through-hole in a 3-d body. For example, consider Figure 1 with the layer shrunk to zero thickness and also the consequent core to a line of puncture through the plane of the paper; the core-line represents the through-hole. The 2-d surface is such that a cut along it renders simply-connected the body with the hole. Additionally, the gradient of the potential field is required to match a prescribed irrotational vector field on the simply-connected domain obtained through the cut.

We note here that the question above is much simpler in its details than the question posed by Weingarten and Volterra related to understanding the jumps of a displacement (vector) field when its symmetrized gradient is required to match a prescribed symmetric tensor field which satisfies the St.-Venant compatibility condition of linear elasticity theory in the simply-connected domain resulting from a planar cut of the multiplyconnected domain with the hole. Indeed, in this latter case, a key construction is a skewsymmetric tensor field (infinitesimal rotation) from the prescribed symmetric tensor field, whose jump across the cut-surfaces (that serve as parts of the boundary of the simplyconnected domain) relates directly to the disclinations of linear elasticity theory. It should be clear, then, that in the liquid crystal theory context, even if one chose to discuss the issue of allowable jumps in the director field context rather than the angle parametrization field, there is no natural way a relevant symmetric tensor field arises from the director gradient whose unique2 infinitesimal rotation field in a simply-connected domain may then be used to define the notion of an isolated singular disclination in liquid crystal theory. Given that the Oseen-Frank and Ericksen-Leslie theories are geometrically exact,

\footnotetext{
${ }^{2} \mathrm{Up}$ to a spatially uniform skew-symmetric tensor field.
} 
the situation is not very different if a link is attempted between liquid crystal disclinations and the singular defects that characterize the jumps in finite rotation associated with an elastic strain field that satisfies the finite strain compatibility conditions in a multiplyconnected domain. Again, the issue crucially hinges on the question being posed in terms of the basic tensor field being a positive-definite symmetric second order tensor field (the Right-Cauchy-Green tensor field), and there does not seem to be any natural way in which the director gradient may be considered to generate such a field. Such an observation is consistent with that made in KF08] (Sec. I.E.1, p. 67): "This explains the interest in reconsidering the continuous theory of defects, although new concepts have to emerge. The case of mesomorphic phases requires an extension of the theory of continuous defects for solids to situations where there is locally only one physical direction (the director), i.e. no local trihedron of directions, as in the uniaxial nematic $N$, the $\operatorname{Sm} A$, and the columnar $D$ cases." We believe that our work provides the natural extension of the theory of continuous defects in solids to the mesomorphic phases named above.

As a further example of such differences, we note, as mentioned in KF08] Sec. IIA (p. 69, Remark), that the Volterra process is properly defined only for magnitude of rotation angles less than $\pi$. However, as far as wedge disclinations in liquid crystals are concerned, the $+\frac{1}{2}$ and $+\frac{3}{2}$ strength defects are entirely different entities; as shown in Section 4, our theory is able to predict such differences.

4. Computation of bounded energy distortion and director solutions. We demonstrate and evaluate the ideas presented in Section 3.2 through explicit constructions for different kinds of defects. First, we present results for straight wedge and twist disclinations and compare our results with Frank's planar model [Fra58]. Then we take the next step forward and simulate twist disclination loops.

4.1. Straight disclinations. We refer to disclinations with core cylinder along a straight line, as opposed to a general curve or a closed loop, as straight disclinations. A straight wedge disclination is one for which the defect line is along the axis of rotation of the director field. In the case of a twist disclination, the line is perpendicular to the axis of rotation.

4.1.1. Wedge disclinations. Although it is uncommon to find an isolated wedge disclination in nature due to its considerable energy cost, it is nevertheless a canonical problem for theoretical tools, and we study such a 2-dimensional line defect [KL03.

Consider $\theta$ as a function of the coordinates $x_{1}$ and $x_{2}$ so that the director field would be planar. According to (8), (11) and Stokes' theorem,

$$
\int \beta_{3}^{\theta} d A=-\int \operatorname{curl} \boldsymbol{\lambda} \cdot \boldsymbol{e}_{3} d A=-\oint \boldsymbol{\lambda} \cdot d r=2 \pi K
$$

This criterion indicates that the line integral of the vector field $\boldsymbol{\lambda}$ over any closed curve bounding the core is non-vanishing. Inspired by the Weingarten-Volterra process in elastic dislocation theory and adapting it to the simpler situation at hand as indicated in Sections 3.2 3.4, we define $\boldsymbol{\lambda}$ as a vector field with support on a thin layer that originates 
from the defect core and extends all the way to the boundary:

$$
\begin{gathered}
\boldsymbol{\lambda}:=-\frac{2 \pi K}{2 r_{1}} \boldsymbol{e}_{1} \quad \text { for }\left\{\begin{array}{l}
x_{2}>0 \\
\left|x_{1}\right|<r_{1},
\end{array}\right. \\
\boldsymbol{\lambda}=\mathbf{0} \text { otherwise. }
\end{gathered}
$$

Here, $r_{1}$ defines the spatial width of the layer. The derivative of $\boldsymbol{\lambda}$ with respect to $x_{2}$ is zero everywhere, except at the layer boundary at $x_{2}=0$. Consequently, $\boldsymbol{\beta}^{\theta}$ will be a singular wall-like distribution of finite extent supported on this layer boundary. However, for reasons indicated in Section 3.2, the total energy of the disclination so defined would still necessarily be finite.

In agreement with Figure 1, we now endow this singular wall with a finite width 3 Thus, the following definition for the layer field is used:

$$
\begin{aligned}
& \lambda_{1}:=\left\{\begin{array}{ll}
\frac{-2 \pi K}{2 r_{1}} & x_{2}>\frac{r_{0}}{2} \\
\frac{-2 \pi K}{2 r_{1}} \frac{r_{0}+2 x_{2}}{2 r_{0}} & \left|x_{2}\right| \leq \frac{r_{0}}{2}
\end{array} \quad\left|x_{1}\right|<r_{1},\right. \\
& \lambda_{1}=0 \text { otherwise, } \\
& \lambda_{2}=0 \text { everywhere. }
\end{aligned}
$$

Figure 2 shows the norm of $\boldsymbol{\lambda}$ and the resulting $\boldsymbol{\beta}^{\theta}$ field from it, where both fields have been normalized by $K$ :

$$
\tilde{\boldsymbol{\lambda}}=\frac{1}{K} \boldsymbol{\lambda} \quad \tilde{\boldsymbol{\beta}}^{\theta}=\frac{1}{K} \boldsymbol{\beta}^{\theta} .
$$

Note that, as mentioned in Section $\underline{3.2}$, the $\boldsymbol{\beta}$ field may not be as localized as $\boldsymbol{\beta}^{\theta}$ since

$$
\begin{aligned}
\boldsymbol{\beta} & =\operatorname{curl}(\boldsymbol{E}), \\
\beta_{i j} & =e_{j k l} E_{i l, k} \\
& =e_{j k l}\left(\frac{\partial n_{i}}{\partial \theta} E_{l}^{\theta}\right)_{, k} \\
& =e_{j k l}\left(\frac{\partial^{2} n_{i}}{\partial \theta^{2}} \theta_{, k} E_{l}^{\theta}+\frac{\partial n_{i}}{\partial \theta} E_{l, k}^{\theta}\right) \\
& =e_{j k l}\left(\frac{\partial^{2} n_{i}}{\partial \theta^{2}} \theta_{, k}\left(\theta_{, l}-\lambda_{l}\right)+\frac{\partial n_{i}}{\partial \theta} E_{l, k}^{\theta}\right),
\end{aligned}
$$

and noting that $e_{j k l} E_{l, k}^{\theta}=\beta_{j}^{\theta}$ and $e_{j k l} \theta_{k} \theta_{l}=0$,

$$
\beta_{i j}=-e_{j k l}\left(\frac{\partial^{2} n_{i}}{\partial \theta^{2}} \theta_{, k} \lambda_{l}\right)+\frac{\partial n_{i}}{\partial \theta} \beta_{j}^{\theta} .
$$

One can easily see that while the second term is localized inside the defect core, the first term is not necessarily zero inside the layer. However, since $\theta_{, k}$ decays sharply from the defect core, the effect of this term vanishes rapidly away from the core. Figure 3 shows this deviation from being a perfectly localized field.

Equations (7), (14), and (18) yield the director field from Poisson's equation

$$
\operatorname{div}(\operatorname{grad} \theta)=\operatorname{div} \boldsymbol{\lambda} \text {. }
$$

\footnotetext{
${ }^{3}$ Of course, ideally one would seek such a width as an outcome of the full dynamical model. As a first step, we show here that isolated disclinations of specified strength with finite-size cores can be solutions to balance of moments in our model.
} 


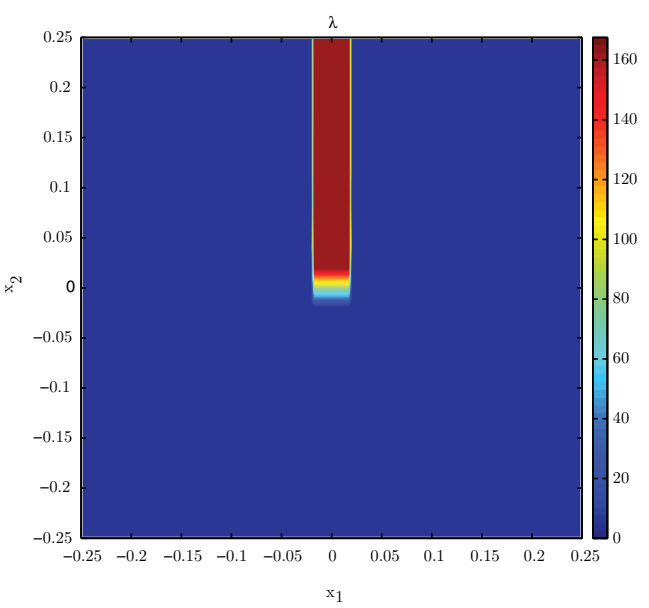

(a) $|\tilde{\lambda}|$

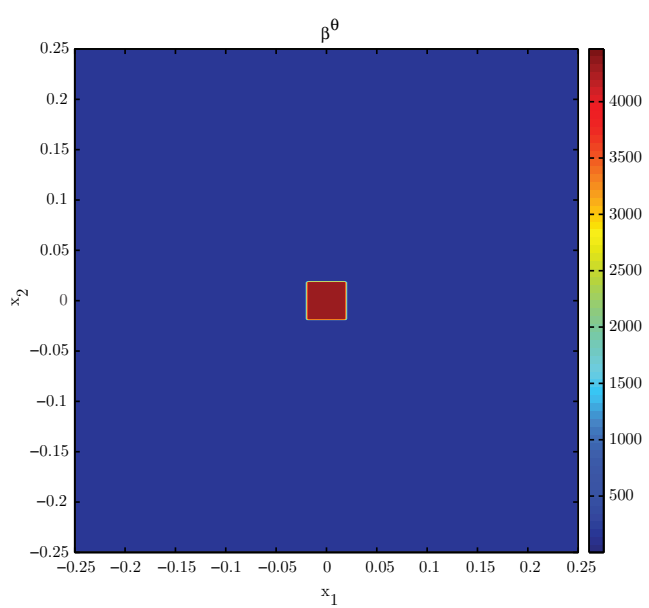

(b) $\left|\tilde{\boldsymbol{\beta}^{\theta}}\right|$

FIG. 2. Normalized $\boldsymbol{\lambda}$ field, resulting from (18), and the corresponding $\boldsymbol{\beta}^{\theta}$ field.

As a close reference point for the numerically computed approximate director solutions that are generated subsequently, the closed-form solution of (20) in an infinite domain is derived in Appendix $\mathrm{A}$ corresponding to smooth approximating sequences to the discontinuous layer fields, i.e. (17)-(18), we consider.

In order to compare our energy and director field results with those available in the literature (e.g. [KL03]), we will consider line wedge disclinations in an infinite space. To compare the results with Frank's planar model, we consider the following equation with Neumann boundary condition:

$$
\left\{\begin{array}{l}
\text { divgrad } \theta=\operatorname{div} \boldsymbol{\lambda} \quad \text { on } V \\
(\operatorname{grad} \theta-\boldsymbol{\lambda}) \cdot \boldsymbol{\nu}=\operatorname{grad} \phi \cdot \boldsymbol{\nu} \text { on } \Gamma,
\end{array}\right.
$$




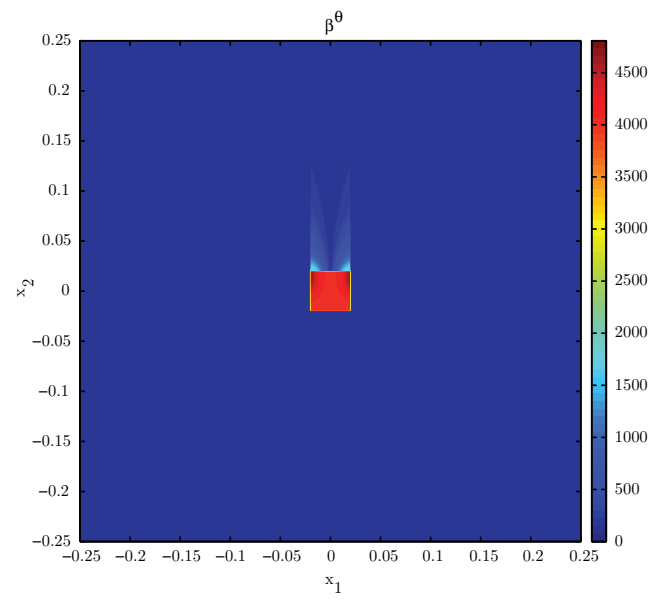

(a) $|\tilde{\boldsymbol{\beta}}|$

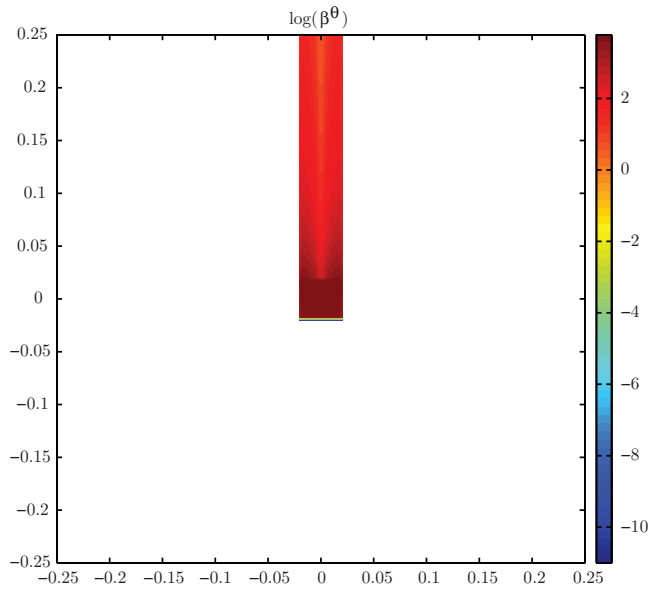

(b) $\log (|\tilde{\boldsymbol{\beta}}|)$

Fig. 3. Normalized $\boldsymbol{\beta}$. The affects of the first term in (19) are more obvious in the logarithmic plot. The white area in part (b) represents zero for $\tilde{\boldsymbol{\beta}}$.

where $\Gamma$ is the exterior boundary of the finite domain $V$ and $\phi$ is the angle of the director field with the $x_{1}$ axis in Frank's solution [Fra58:

$$
\phi=K \tan ^{-1}\left(\frac{x_{2}}{x_{1}}\right)+c
$$

with $c$ a constant. For the purpose of evaluating the boundary condition for the domains involved, it suffices to consider $\operatorname{grad} \phi$ to be given by

$$
\frac{K}{r^{2}}\left(-x_{2} e_{1}+x_{1} e_{2}\right)
$$


(to the neglect of a surface Dirac distribution related to the discontinuity in Frank's solution (21)). Moreover, one must assign the value of $\theta$ at one point in the domain for uniqueness of solutions. Note that this assignment is tantamount to fixing $c$ in Frank's solution. We choose to assign a value $\theta_{0}$ at one point on the right-hand-side boundary of the layer/strip on which $\boldsymbol{\lambda}$ has support.

Now, using this Neumann boundary condition, the weak form of (20) for use in the Galerkin finite element method is

$$
\int_{V} \delta \theta_{, i}\left(\theta_{, i}-\lambda_{i}\right) d V-\int_{\Gamma} \delta \theta \phi_{, i} \nu_{i} d S=0 .
$$

Here $\delta \theta$ is a continuous test function for $\theta$. Bilinear, 4-node, quadrilateral elements are used for our simulations. Needless to say, the resulting $\theta$ will be a continuous field, and we show converged results for the director field with mesh refinement in Figure 15(b).

Figure 4 shows the result of solving (23) for $\theta$, plotted as director fields using (4), for different disclination strengths. In all of these examples, $\theta_{0}=\frac{\pi}{2}$, except in Figure 4(d). These director fields are completely analogous to those from Frank's solution (21), outside the layer.

However, inside the strip, the continuous $\theta$ field has to compensate the jump between the two sides of the layer. As a result, one can see that inside the layer, directors rotate sharply from one side to the other but at no extra energy cost in the layer beyond what is involved in the standard interpretation of the energy due to Frank's solution, i.e. the energy arising from considering the director gradient field to be (22). This is due to compensation by the $\boldsymbol{\lambda}$ field, more precisely, by the grad $z$ part of the $\boldsymbol{\lambda}$ field. Moreover, the topological content of the standard interpretation, i.e. (22), of the singular gradient of Frank's solution (21) is preserved in the $\boldsymbol{E}^{\theta}$ field and approximately in the gradient of continuous $\theta$ field (up to the layer). Within this setting, even in the case of an integer defect, although there is no apparent discontinuity between the directors on the left and right sides of the layer, there exists a $2 \pi$ transition within the layer. In order to have a better understanding of this compensation, we take a closer look at the case $K=\frac{1}{2}$. Figure [5] shows the rotation of the director field inside the layer. Note that the rotation of the director field inside the layer is opposite in sense to its rotation outside the field.

Next, we compare the energy density field of our theory to the corresponding one of Frank's model. Using (5), the energy term for Frank's planar model will be

$$
\psi_{F}(\boldsymbol{n}, \boldsymbol{E})=\frac{\kappa}{2} \frac{K^{2}}{r^{2}}
$$

(using the standard interpretation of the gradient). Evidently, the total energy in a finite body for such an energy density field will not be finite. However, the energy density of the defects we model is non-singular in most cases and definitely integrable in all cases, so that the total energy will be finite. The energy density of our model can be calculated from the following expression:

$$
\psi(\boldsymbol{n}, \boldsymbol{E})=\frac{1}{2} \kappa\left(\theta_{, i}-\lambda_{i}\right)\left(\theta_{, i}-\lambda_{i}\right)
$$




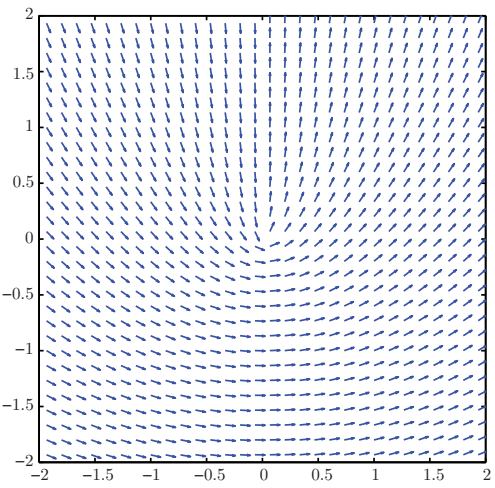

(a) $K=\frac{1}{2}$

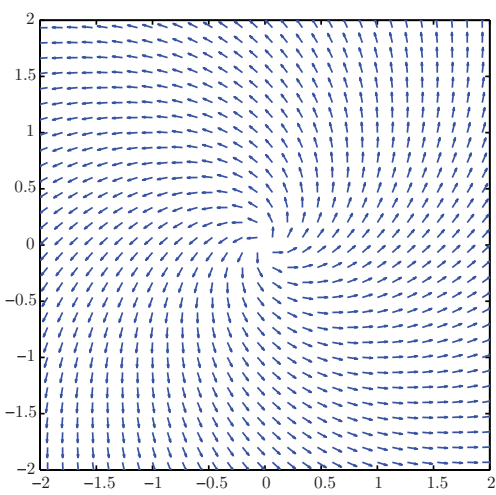

(d) $K=1, c=\pi / 4$

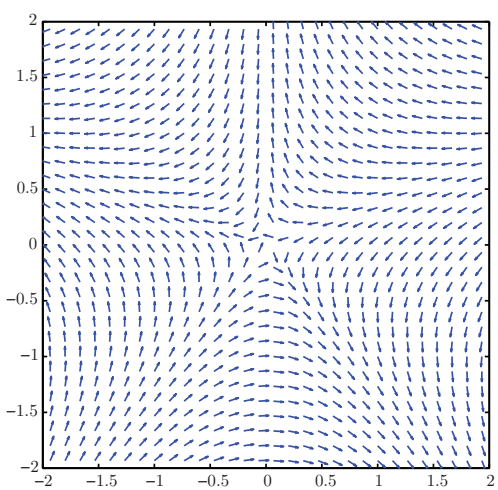

(g) $K=-\frac{3}{2}$

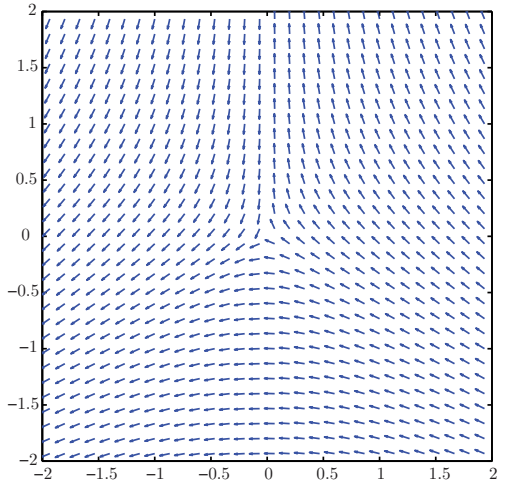

(b) $K=-\frac{1}{2}$

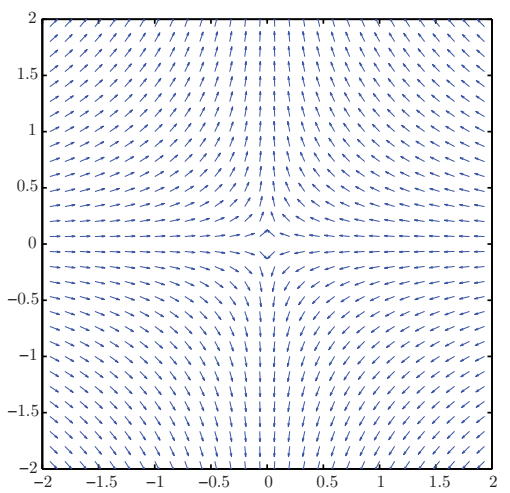

(e) $K=-1$

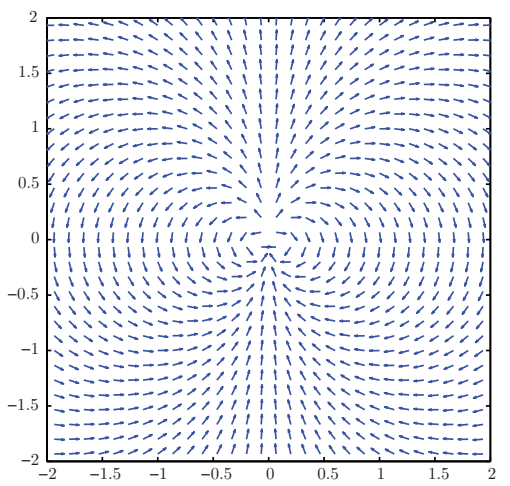

(h) $K=2$

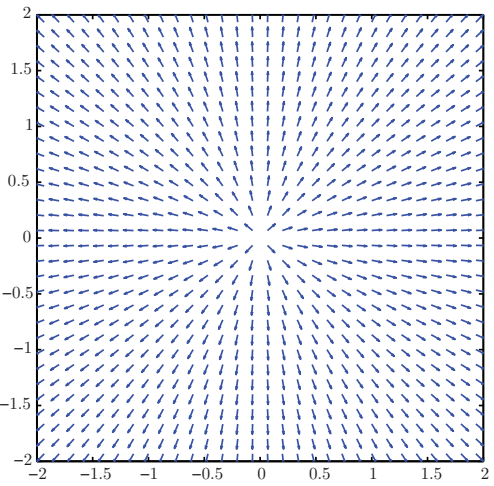

(c) $K=1, c=0$

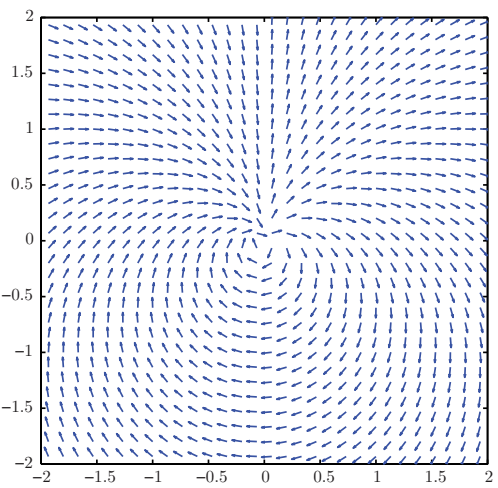

(f) $K=\frac{3}{2}$

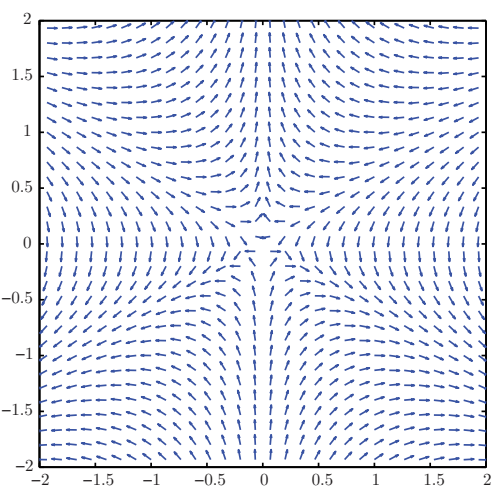

(i) $K=-2$

FIG. 4. Computed director fields with wedge disclination at the center for different disclination strengths. Parts (c) and (d) have the same disclination strength but different prescribed values $\theta_{0}$. 


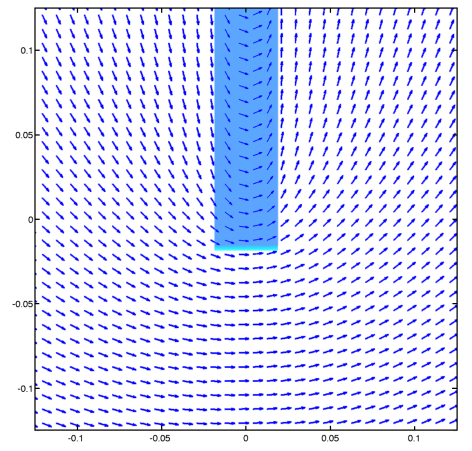

FIG. 5. A closer look at the computed director field for $K=\frac{1}{2}$. The colored strip depicts the layer field, $\boldsymbol{\lambda}$. If one makes a clockwise circuit around the defect core, the rotation of the directors will be clockwise, outside the layer, while it is anti-clockwise inside.

Figure 6] shows energy contours normalized with respect to $K$ and $\kappa$, outside the defect core:

$$
\tilde{\psi_{F}}=\frac{1}{\kappa K^{2}} \psi_{F}=\frac{1}{2 r^{2}} \quad \tilde{\psi}=\frac{1}{\kappa K^{2}} \psi .
$$

In this figure, we have also compared the decay of the energy density as it moves away from the defect core. According to $\psi_{F}$, such decay must follow a $\frac{1}{r^{2}}$ trend. The results from our setting shows good agreement with the analytical results outside the core while being non-singular inside; see Figure 6 .

Note that not only is the energy density in our model non-singular at the center, but Figure 6(b) shows that the energy density profile drops dramatically at the center of the defect core. Later, in Figure 14(b) we will show that the energy level at this point converges to zero. However, this is not a general rule. In fact the energy density profile inside the core depends on the distribution of the layer field, $\boldsymbol{\lambda}$, and consequently $\boldsymbol{\beta}^{\boldsymbol{\theta}}$. For instance, if we cut off the layer field at the center of the defect core instead of letting it decay linearly, i.e. using (17) for the layer field definition, the energy density will be as in Figure 7 . Figure 7(b) reveals the fact that although such sharp cutoff in the layer field will cause unsymmetric energy density distribution inside the defect core, the energy density will still be radially symmetric outside the core.

We also note that even though in this case we have a singular wall-like distribution of $\boldsymbol{\beta}^{\theta}$ at the core edge that can potentially cause singularities in the energy density at the sharp corners defining the layer, the total energy for a fixed width of layer will always converge as long as $r_{1}>0$; i.e. the layer has finite width. This is borne out in our computations where the total energy corresponding to Figure 7 appears to converge, as shown in Figure 8.

4.1.2. Straight twist disclination. Twist disclinations have the axis of rotation of the director field perpendicular to the defect line. They are sometimes called perpendicular disclinations.

From the mathematical point of view, we use the same definition for the planar director field as in equation (4). While the $\boldsymbol{n}$ field lies in the $x_{1}-x_{2}$ (horizontal) plane, $\theta$ is assumed to be a function of $x_{1}$ and $x_{3}$ and it is constant along the $x_{2}$ direction. The 


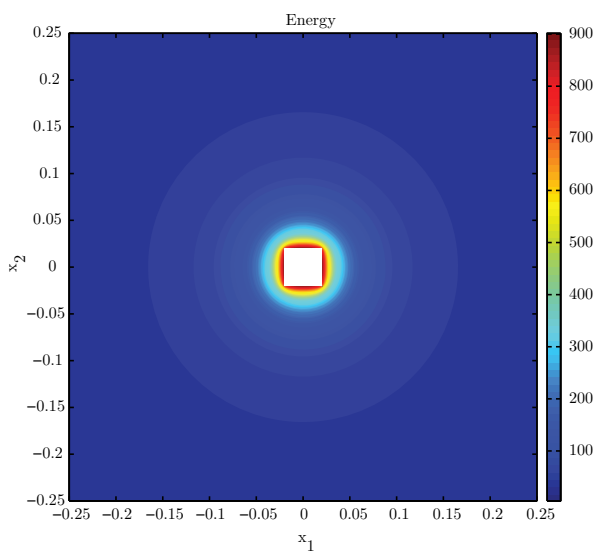

(a)

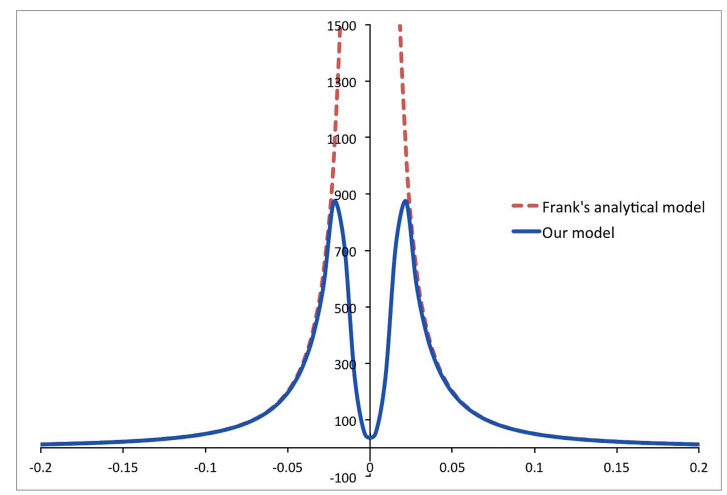

(b)

FIG. 6. (a) Normalized energy density contour outside the defect core and (b) the decay of energy density when $x_{2}=0$.

definition of the layer field undergoes similar, appropriate changes. We define $\boldsymbol{\lambda}$ as in (17), but now in the $\boldsymbol{e}_{3}$ direction instead of $\boldsymbol{e}_{1}$ (for direction of $\boldsymbol{\lambda}$ and the width of the layer), as shown in Figure 9(a). We perform a 3 -d finite element computation in this case. The computed energy density concentration is shown in Figure 9(b). The distribution of the energy and its decay on the vertical plane are exactly the same as shown in Figure 6 for the straight wedge disclinations, which is consistent with expectation. Figures 9(c) and $9(\mathrm{~d})$, respectively, show the computed and analytical director fields.

4.2. Twist disclination loops. Disclination loops are more commonly found in nature than curves terminating at boundaries HR06. In this section we will simulate a twist disinclination loop using our framework.

The director field will be planar, but now the $\theta$ field will be a function of $x_{1}, x_{2}$ and $x_{3}$. The $\boldsymbol{\lambda}$ field for a planar twist disclination loop is taken to be a constant field in a bounded layer, as can be seen in Figure 10. The bounding cylinder of the layer over 


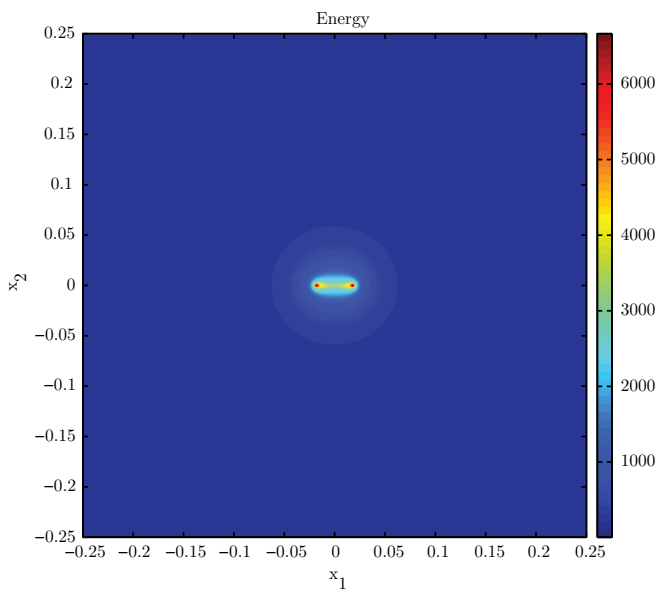

(a)

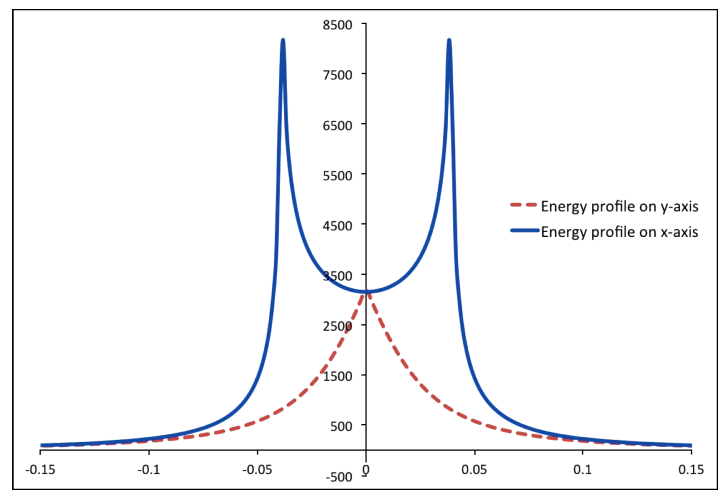

(b)

FIG. 7. (a) Normalized energy density contour when $\boldsymbol{\lambda}$ sharply ends at the center, (b) the corresponding energy density profile in cases $x_{2}=0$ (blue, solid line) and $x_{1}=0$ (red, dashed line).

which the constant field decays to zero then forms the disclination loop. Clearly, the closed loop can be of any shape, but restricted to be planar in this exercise.

In particular, we have chosen a square-shaped and a circular loop in the horizontal plane. $\boldsymbol{\beta}$ and the $\boldsymbol{\beta}^{\theta}$ field for the rectangular loop are shown in Figure 11. Just like the 2 -dimensional wedge disclination case, the $\boldsymbol{\beta}^{\theta}$ field is non-zero only at the defect core, which appears as a square loop, and $\boldsymbol{\beta}$ is non-zero inside the loop, where the layer field is non-zero.

The director field is a planar vector field with twist axis along the $x_{3}$ direction. As shown in Figure 12, in any given plane $x_{3}=$ constant the effect of the loop decays rapidly with distance from the loop. Figure 12 also clarifies the way in which the director vectors make a twist as one moves through a circuit enclosing the core. Note that the director vectors corresponding to the lower part of the $\boldsymbol{\lambda}$ field have the opposite direction of the 


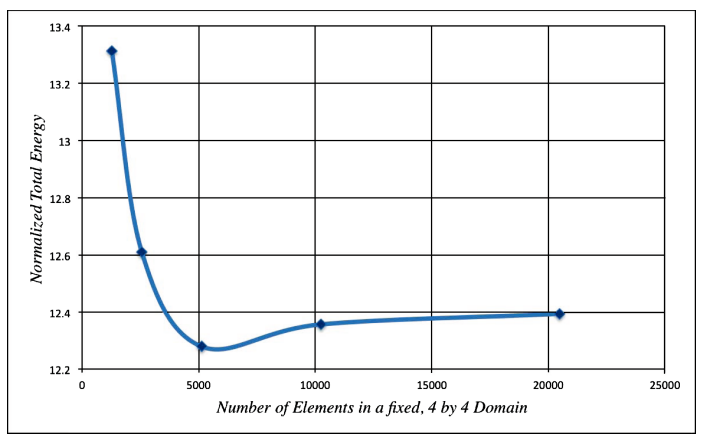

FIG. 8. Convergence of the normalized total energy corresponding to the energy density shown in Figure 7 with the increase of the number of elements used in a fixed domain.

ones on the top, since that is a half-integer disclination loop $\left(K=\frac{1}{2}\right)$ and as a result the director field makes a $\pi$-radian twist. We emphasize that while this twist deficit is observable in the (continuous) director field only on circuits that are 'closed up to the thickness of the core', integrating $\boldsymbol{E}^{\theta}$ on an exactly closed circuit encircling an arm of the disclination loop core would yield a $\pi$-radian twist by design.

Figure 13 shows the energy plots of the two disclination loops from different points of view. Figures $13(\mathrm{a})$ and $13(\mathrm{~b})$ are the energy density contours at $x_{3}=0$ on the $x_{1}-x_{2}$ plane for the square-shaped and circular loop, respectively. Note that the energy density of the rectangular loop has significantly higher magnitude at the corners, this being a consequence of the high curvature in the director field at the corners of the loop. Similarly to what we saw in Figure 6(b) these contour plots show a drop in magnitude inside the core. This plot also shows smoothness of the energy density, even in the vicinity of the defect core. In order to better understand the pattern of the energy density on this plane, Figures 13(c) and 13(d) show the logarithmic scale of the plot. A closer look at these two plots reveals that even for the rectangular defect core the energy density adopts a radially symmetric patter away from the core. The observable deviation from this circular texture on the boundary is due to the zero Neumann boundary condition that we have used for these two examples. Figures $13(\mathrm{e})$ and $13(\mathrm{f})$ show the logarithmic plot of the energy density on the $x_{1}-x_{3}$ plane.

4.3. Convergence of numerical results. One of the main advantages of our model is the possibility of attaining non-singular results in energy density. In this section we have verified this claim by refining the mesh in our numerical model to demonstrate convergence even inside the defect core. Figure 14 shows this convergence for the straight wedge disclination. Since the results for different disclination strengths are similar, we have normalized our energy plots with respect to $K$.

The energy density results for our model and Frank's analytical model are the same outside the core. Figure 15 shows a relative error of less than $1 \%$ everywhere for the energy density, and less than $0.6 \%$ for the director field (outside the layer) for the halfinteger defect. Results for all other disclination strengths show the same error distribution. 


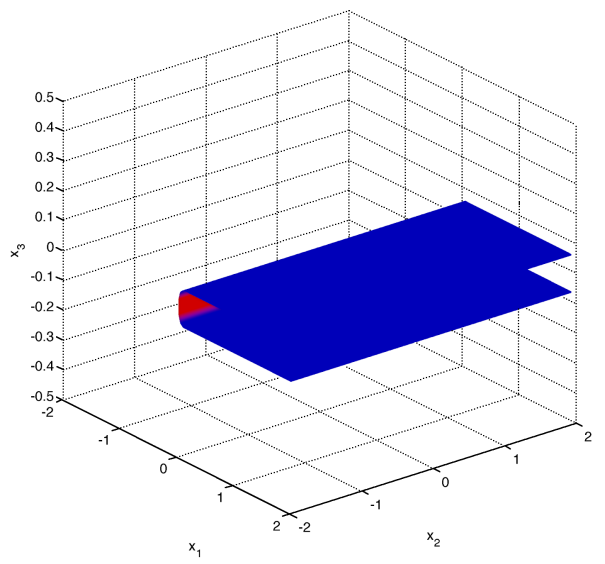

(a)

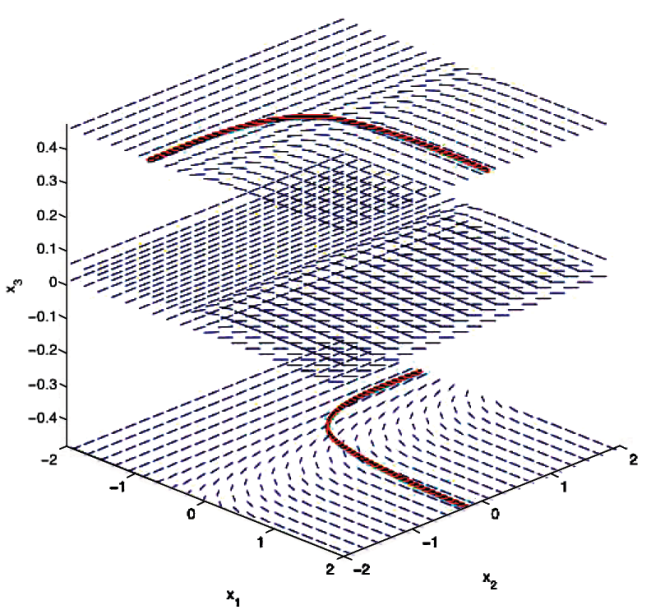

(c)

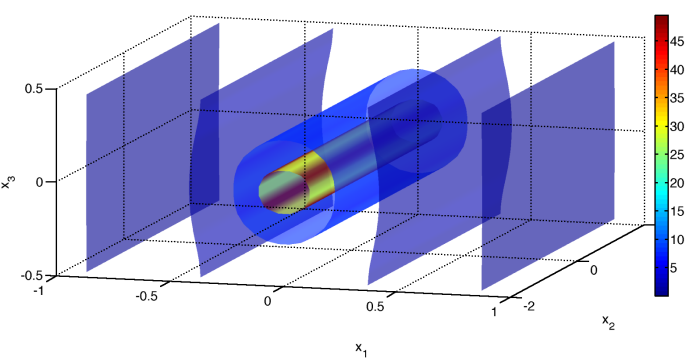

(b)

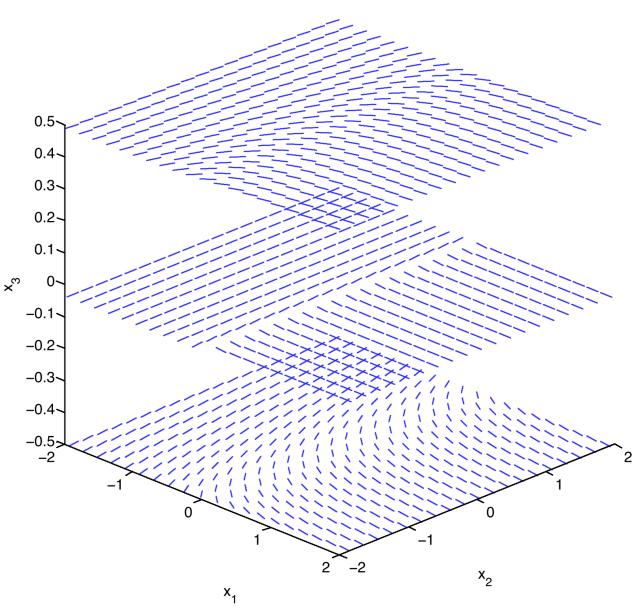

(d)

FIG. 9. Straight twist disclination: (a) 3D plot of the layer field, $\boldsymbol{\lambda}$, (b) a 3D plot of different layers of the normalized energy density to illustrate the energy density distribution in the body, (c) is the computed director field, while (d) is the analytical one.

Finally, Figure 16] shows the convergence of the energy density in the square-shaped twist loop model. In this figure, the energy density profile of the loop with two different element sizes has been compared on the $x_{1}$ axis, i.e. on the line $x_{2}=0, x_{3}=0$.

5. Balance of forces. Assuming a single disclination in the body (loop or a core cylinder terminating at the boundaries of the body), static balance of linear momentum (2) is satisfied in the region of the body excluding the core. This is so since the stress tensor is given by

$$
T_{i j}=-p \delta_{i j}-\kappa E_{i}^{\theta} E_{j}^{\theta}
$$




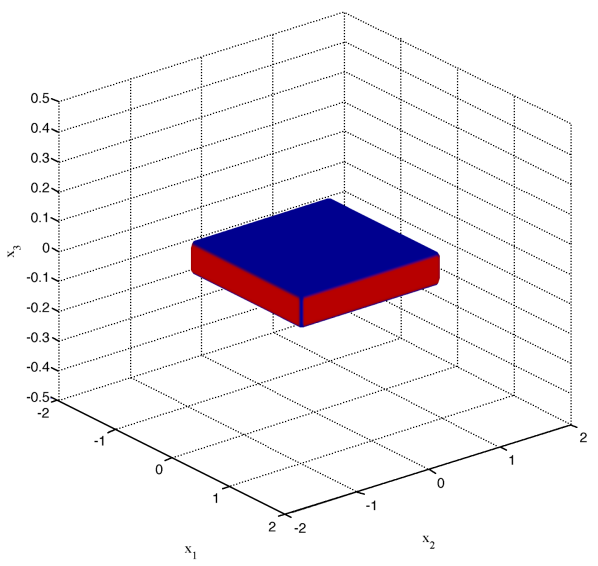

FIG. 10. An example of $\boldsymbol{\lambda}$ field for a square-shaped twist disclination loop. The field vanishes in the white region.

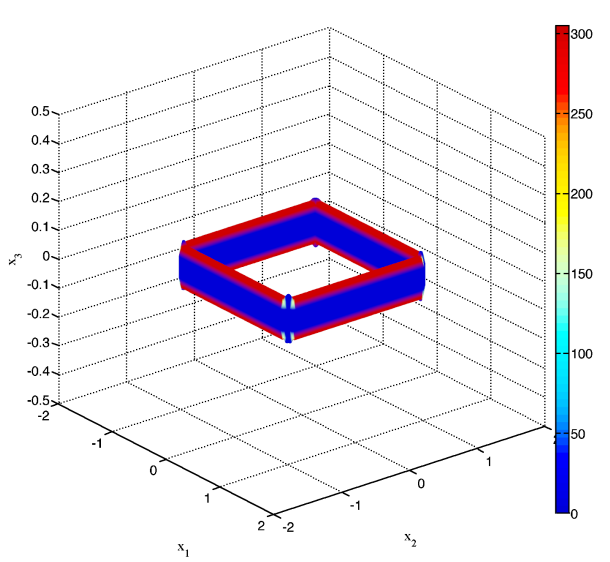

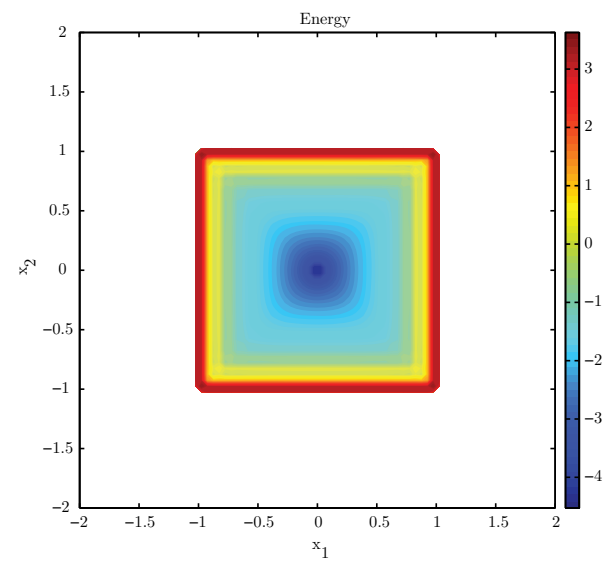

(a)

FIG. 11. (a) Normalized $\boldsymbol{\beta}^{\theta}$ for the twist disclination loop and (b) logarithmic contour plot of normalized $|\boldsymbol{\beta}|$. The white area in part (b) represents zero for $|\boldsymbol{\beta}|$.

and (77) and (9) imply, along with the core-support of $\boldsymbol{\beta}^{\theta}$, that

$$
\left(E_{i}^{\theta} E_{j}^{\theta}\right)_{, j}=\left(E_{j}^{\theta} E_{j}^{\theta}\right)_{, i}=\frac{\psi_{, i}}{\kappa},
$$

and one chooses the indeterminate constraint pressure field in the excluded-core region of the body as

$$
p=-\psi
$$

to satisfy (2). On the external boundary of the body, recognizing (10) one chooses $p=0$. Thus, the resultant force on the surface of the core cylinder (or any other surface enveloping it) must vanish. 


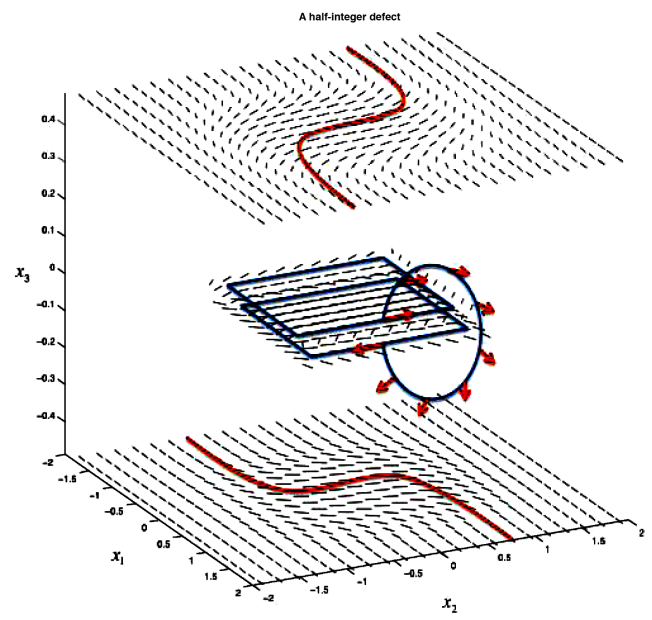

(a) Square-shaped loop

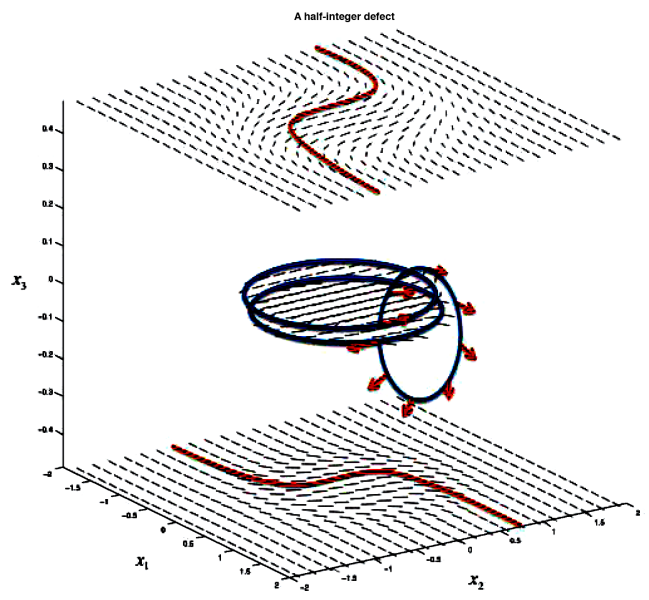

(b) Circular loop

FIG. 12. Computed director fields of twist disclination loops for $K=\frac{1}{2}$.

An interesting possibility is afforded by the model AD12 in satisfying static balance of linear momentum everywhere. Note that within the core $\operatorname{curl} \boldsymbol{E}^{\theta}$ does not vanish so that it is not a priori clear that $\left(E_{i}^{\theta} E_{j}^{\theta}\right)_{, j}$ can be expressed as the gradient of a scalar field. However, the vanishing of the curl of the field $\left(\operatorname{grad} \boldsymbol{E}^{\theta}\right) \boldsymbol{E}^{\theta}$ along with the conditions (7][8] 9]) within the core region may be imposed as a design constraint on the $\boldsymbol{\beta}^{\theta}$ field. Were solutions to this system for $\boldsymbol{\beta}^{\theta}$ to exist, one could proceed to set up the field $\boldsymbol{\lambda}$ as before with the caveat that now the field $\operatorname{curl} \boldsymbol{\lambda}$ within the core is not subject to the mild constraint (8) but instead to the pointwise constraint defined by our calculated $\boldsymbol{\beta}^{\theta}$ field. 
Alternatively, the theory also allows the ansatz

$$
\boldsymbol{E}=\sum_{\alpha=1}^{2} \frac{\partial \boldsymbol{n}}{\partial \theta^{\alpha}} \otimes\left[\operatorname{grad} \theta^{\alpha}-\boldsymbol{\lambda}^{\alpha}\right],
$$

and choosing this affords four more degrees of field degrees of freedom to satisfy static balance of linear momentum without body forces simultaneously with satisfying balance of angular momentum, presumably even without necessarily requiring the constraint of incompressibility. Moreover, the solutions generated in this paper would seem to suggest that these extra degrees of freedom could be invoked only in the core cylinder, thus conferring an automatic multiple spatial length scale feature to the theory. Of course, with static balance of linear momentum satisfied everywhere (including cores), the resultant force of the Ericksen stress tensor (and hence the energy-momentum tensor of the classical nematic theory Esh80, CF02]) on the bounding surface enveloping any segment of a core has to vanish without the requirement of any externally applied body forces or the onset of flow, while the AD12 dissipative dynamical theory provides a driving force for disclination motion not related to the Ericksen stress or its resultant. This possibility has the potential of reconciling several different points of view and clarifying an often confusing debate in the liquid crystal literature (cf. [Esh80, Krö93, Eri95]) revolving around the physically meaningful question of modeling the experimentally observed motion of disclination curves in the presence of negligible or no flow.

6. Concluding remarks. We have demonstrated the possibility of representing nonsingular isolated disclination fields within a dynamic theory AD12 of liquid crystalline media designed to account for disclination and dislocation defects. The solutions satisfy balance of moments everywhere in the body, including the core. Balance of forces is satisfied outside the cores, and it appears possible, by going beyond the ansatz (3) assumed in this work, to satisfy force balance everywhere in the body. The fields constructed here may be considered to be equilibria for the theory when the defect lines are considered to be externally pinned (i.e. the defect velocity field, a constitutive quantity, is assumed to vanish).

Our primary goal has been to demonstrate distinguished pairs of director and (incompatible) director distortion fields $(\boldsymbol{n}, \boldsymbol{E})$ satisfying (11) and (9) that generate classically familiar director and energy density fields of disclinations (but not exactly the same ones). In doing so, we use an intermediary 'layer' field which, thankfully, is not required in the subsequent dynamics of the $\mathrm{AD} 12$ model once the director and the director distortion fields have been constructed. However, a fact that gives us pause is that the director incompatibility tensor fields $\boldsymbol{\beta}$ of these isolated disclinations appear to contain some signature of the layer field used to construct them even outside the region that one would typically like to associate with the core of the disclination, and whether this is physically realistic or not remains to be seen. Also, the director fields that we construct have a rapid variation in the layer (without a corresponding energy cost), and the physical realism of such a distribution should also be testable in the modeling of situations involving interaction of the director field with flow. Of course, the analysis presented in Section 3.2 provides the logic to construct a director and director incompatibility field 
without the layer-related features discussed above. To wit, considering the example of the axial wedge disclination of Section 4.1 in an infinite domain, we define

$$
\boldsymbol{\lambda}_{s}=\operatorname{grad} z_{s}+\boldsymbol{E}_{r}^{\theta}
$$

where

$$
\operatorname{div}\left(\operatorname{grad} z_{s}\right)=\operatorname{div} \tilde{\boldsymbol{\lambda}}
$$

and $\tilde{\boldsymbol{\lambda}}$ is the distribution obtained in (17) by letting $r_{1} \rightarrow 0$. This results in $z_{s}$ being exactly Frank's solution (21). Additionally, we let $\boldsymbol{E}_{r}^{\theta}$ be the solution to

$$
\begin{aligned}
\operatorname{div} \boldsymbol{E}_{r}^{\theta} & =0 \\
\operatorname{curl} \boldsymbol{E}_{r}^{\theta} & =\boldsymbol{\beta}_{r}^{\theta}
\end{aligned}
$$

(with natural decay requirements at large distances) where $\boldsymbol{\beta}_{r}^{\theta}$ is a radially symmetric core function described in Appendix B with associated solution for $\boldsymbol{E}_{r}^{\theta}$. This formula shows that outside the core, $\boldsymbol{E}_{r}^{\theta}$ matches the standard interpretation (22) of the gradient of Frank's solution (21). Setting $\boldsymbol{E}^{\theta}:=\operatorname{grad} \theta-\boldsymbol{\lambda}_{s}$ and requiring it to satisfy the governing equations of our model, i.e. (77) and (9), a (discontinuous) solution is certainly $\theta=z_{s}$, with discontinuity supported on the half-plane defined by $r_{1} \rightarrow 0$ in the definition (17) of $\tilde{\boldsymbol{\lambda}}$. These results imply that outside the core and away from the half-plane $x_{1}=0, x_{2}>0$, $\boldsymbol{E}_{r}^{\theta}$ matches grad $\theta$. Assuming the validity of (15) on the half-plane and noting that the $\operatorname{grad} \theta$ and $\boldsymbol{E}^{\theta}$ fields are parallel to $\boldsymbol{e}_{1}$ on it, it may be concluded that $\boldsymbol{\beta}$ is non-vanishing only inside the core. With $\boldsymbol{E}^{\theta}$ defined as in this paragraph, we note that even though $\operatorname{grad} \theta$ is not a locally integrable field, this is exactly compensated by $\operatorname{grad} z_{s}$ so that the energy density is formed from the field $\boldsymbol{E}_{r}^{\theta}$ alone. In this connection, we note the interesting fact that the classical defect theory works with a director-'gradient' field for forces, moments and energetics that is not strictly a generalized derivative of what it states as the director field (even outside the core) 4 Thus, it would seem that considering the energy formed from a director distortion field distinct from the director gradient in the presence of line defects (as we do) is probably a reasonable logical device even for discussing the classical theory.

This paper has not made any statements about whether the chosen isolated defectlike director and director distortion fields can actually occur as equilibria of the overall dynamical model AD12 for suitable constitutive assumptions for defect velocities (e.g. a linear kinetic assumption on disclination velocity as a function of its theoretical driving force). Based on our experience with related work on Field Dislocation Mechanics Ach10, AT11, AMZ10, we believe this should be possible with the use of non-convexity in OZF energy (ossibly due to a limit on director elasticity), the system

\footnotetext{
${ }^{4}$ The analog of Weingarten's construction to be utilized here makes it entirely clear why this must be so: there, one begins with an irrotational field in a doubly-connected 3-d domain with a through-hole, and what may be associated as the 'closest' potential field generating the irrotational field is not defined on the same domain but rather on a simply-connected domain obtained through a single cut of the doubly-connected domain that renders the latter simply-connected. Unfortunately, however, these are not procedures that lend themselves to practical nonlinear theory, especially of the type interpretable by the intelligence level of electronic computers that are essential to probe the complex dynamics of the theories we have in mind.
} 
(as opposed to scalar) nature of the defect evolution equations, as well as from appropriate boundary conditions. In the presence of flow, there are, of course, other natural means to induce line defects.

There are many natural further steps to explore: solving for more-than-planar fields of isolated defects, defect solutions for the full 4-constant Oseen-Frank energy, solutions for disclinations coupled to dislocations in smectics and columnar phases and on to dynamics with and without flow (without flow and even without director inertia, the model has dynamics due to defect evolution). While each of these problems is significant in its own right, we take some satisfaction in the fact that all of these questions can at least be posed unambiguously within our modeling framework. Finally, an important future task is to make contact with the fine topological work of Kleman and Friedel [KF08] and Kamien and co-workers CAK09].

\section{Appendices.}

Appendix A. An explicit solution for $\theta$. An analytic solution for the director field corresponding to $\boldsymbol{\lambda}$ source fields in (20) in an infinite domain is developed to serve as motivation for some of our claims in Section 3.2 The source fields are layer-like but do not have compact support on bounded domains. Consider a $\delta$ sequence [Han06]:

$$
\delta_{n}(x)=\frac{n}{\sqrt{\pi}} e^{-(n x)^{2}},
$$

so that $\int_{-\infty}^{\infty} \delta_{n}(x)=\operatorname{erf}(x)=1$, for any $n$. Also, consider an $H$ sequence, which converges to the Heaviside step function

$$
H_{n}(x)=\frac{1}{2}\left(\tan ^{-1}(n x)+1\right) .
$$

Now, one can define $\boldsymbol{\lambda}$ as

$$
\boldsymbol{\lambda}_{n}(\boldsymbol{x})=2 \pi K \delta_{n}\left(x_{1}\right) H_{n}\left(x_{2}\right) \boldsymbol{e}_{1} .
$$

Note that, for any given $n$,

$$
\oint \boldsymbol{\lambda}_{n} \cdot d r=2 \pi K \int_{x_{1}^{+}} \delta_{n}\left(x_{1}\right) d x_{1}=2 \pi K .
$$

In order to solve (20), one can use Green's function method for an infinite 2-D domain, where the Green function is

$$
G\left(\boldsymbol{x}, \boldsymbol{x}^{\prime}\right)=\frac{1}{2 \pi} \ln \left(\left|\boldsymbol{x}-\boldsymbol{x}^{\prime}\right|\right) .
$$

Now, the expression for $\theta_{n}$ in terms of the Green function will be

$$
\theta_{n}=\int_{V^{\prime}} G\left(\boldsymbol{x}, \boldsymbol{x}^{\prime}\right) \operatorname{div} \boldsymbol{\lambda}_{n}\left(\boldsymbol{x}^{\prime}\right) d V .
$$


Integrating by part and considering the fact that the $\boldsymbol{\lambda}_{n}$ field has only the $\boldsymbol{e}_{1}$ component, we get

$$
\begin{aligned}
\theta_{n} & =\int_{\partial V^{\prime}} G\left(\boldsymbol{x}, \boldsymbol{x}^{\prime}\right) \boldsymbol{\lambda}_{n}\left(\boldsymbol{x}^{\prime}\right) \cdot \boldsymbol{\nu} d S-\int_{V^{\prime}} \frac{\partial}{\partial x_{1}^{\prime}} G\left(\boldsymbol{x}, \boldsymbol{x}^{\prime}\right) \boldsymbol{\lambda}_{n}\left(\boldsymbol{x}^{\prime}\right) d V \\
& =-\frac{1}{2 \pi} \int_{V^{\prime}} \frac{x_{1}^{\prime}-x_{1}}{\left|\boldsymbol{x}^{\prime}-\boldsymbol{x}\right|^{2}} \boldsymbol{\lambda}_{n}\left(\boldsymbol{x}^{\prime}\right) d V \\
& =\frac{1}{2 \pi} \int_{x_{1}^{\prime}} \int_{x_{2}^{\prime}} \frac{x_{1}-x_{1}^{\prime}}{\left|\mathbf{x}^{\prime}-\mathbf{x}\right|^{2}} 2 \pi k \delta_{n}\left(x_{1}^{\prime}\right) H_{n}\left(x_{2}^{\prime}\right) d x_{2}^{\prime} d x_{1}^{\prime}
\end{aligned}
$$

As a result,

$$
\theta_{n}=\frac{K n}{2 \sqrt{\pi}} \int_{x_{1}^{\prime}} \int_{x_{2}^{\prime}} \frac{x_{1}-x_{1}^{\prime}}{\left|\boldsymbol{x}^{\prime}-\boldsymbol{x}\right|^{2}} e^{-\left(n x_{1}^{\prime}\right)^{2}}\left(\tan ^{-1}\left(n x_{2}^{\prime}\right)+1\right) d x_{2}^{\prime} d x_{1}^{\prime} .
$$

Note that in case of $n \rightarrow \infty$,

$$
\begin{aligned}
\theta_{\infty} & =\frac{K}{2} \int_{x_{1}^{\prime}} \int_{x_{2}^{\prime}} \frac{x_{1}-x_{1}^{\prime}}{\left|\boldsymbol{x}^{\prime}-\boldsymbol{x}\right|^{2}} \delta_{\infty}\left(x_{1}^{\prime}\right) H\left(x_{2}^{\prime}\right) d x_{2}^{\prime} d x_{1}^{\prime} \\
& =\frac{K}{2} \int_{x_{2}^{\prime}=0}^{\infty} \frac{x_{1}}{x_{1}^{2}+\left(x_{2}-x_{2}^{\prime}\right)^{2}} d x_{2}^{\prime} \\
& =\left.\frac{K}{2} \tan ^{-1}\left(\frac{x_{2}-x_{2}^{\prime}}{x_{1}}\right)\right|_{x_{2}^{\prime}=0} ^{\infty} .
\end{aligned}
$$

As a result, $\theta_{n}$ will converge to

$$
\theta=K \tan ^{-1}\left(\frac{x_{2}}{x_{1}}\right)+c
$$

which is exactly Frank's solution for planar director fields for straight disclinations for the 1-constant energy.

Appendix B. Solution for $E_{r}^{\theta}$. We provide the following solution from AD12 for completeness. Consider $\boldsymbol{\beta}_{r}^{\theta}$ given by

$$
\boldsymbol{\beta}_{r}^{\theta}\left(x_{1}, x_{2}\right)=\left\{\begin{array}{l}
\rho(r) \boldsymbol{e}_{3}, r<r_{c} \\
\mathbf{0}, r \geq r_{c}
\end{array} ; r=\left(x_{1}^{2}+x_{2}^{2}\right)^{\frac{1}{2}}\right.
$$

with the stipulation that

$$
2 K \pi=\int_{0}^{2 \pi} \int_{0}^{r_{c}} \rho(r) d r r d \psi \Rightarrow K=\int_{0}^{r_{c}} \rho(r) r d r
$$

where $K$ is the strength of the disclination and $r_{c}$ is a core radius. Then following Section 5.2 in Ach01, we have the solution

$$
\begin{aligned}
& \text { For } r<r_{c}: \quad E_{r 1}^{\theta}=\frac{-x_{2}}{r^{2}} \int_{0}^{r} \rho(s) s d s, \quad E_{r 2}^{\theta}=\frac{x_{1}}{r^{2}} \int_{0}^{r} \rho(s) s d s . \\
& \text { For } r \geq r_{c}: \quad E_{r 1}^{\theta}=K \frac{-x_{2}}{r^{2}}, \quad E_{r 2}^{\theta}=K \frac{x_{1}}{r^{2}} .
\end{aligned}
$$


It can be checked that this solution indeed satisfies the governing equations for $\boldsymbol{E}_{r}^{\theta}$ for any $\rho$ satisfying the given conditions. For the choice

$$
\rho(r)=\left\{\begin{array}{l}
\frac{2 K}{r_{c}}\left(\frac{1}{r}-\frac{1}{r_{c}}\right), r \leq r_{c} \\
0, r>r_{c}
\end{array}\right.
$$

the inside-core solution evaluates to

$$
\text { For } r \leq r_{c}: \quad E_{r 1}^{\theta}=\frac{-x_{2}}{r^{2}}\left(\frac{2 K}{r_{c}}\left[r-\frac{r^{2}}{2 r_{c}}\right]\right), \quad E_{r 2}^{\theta}=\frac{x_{1}}{r^{2}}\left(\frac{2 K}{r_{c}}\left[r-\frac{r^{2}}{2 r_{c}}\right]\right),
$$

while the outside-core distribution remains unchanged. 


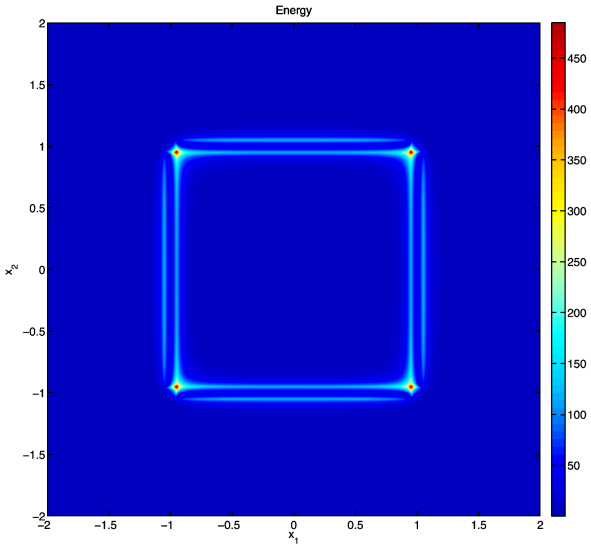

(a)

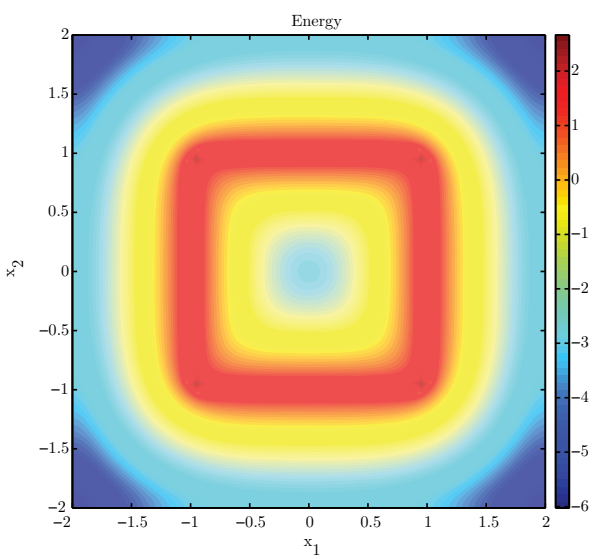

(c)

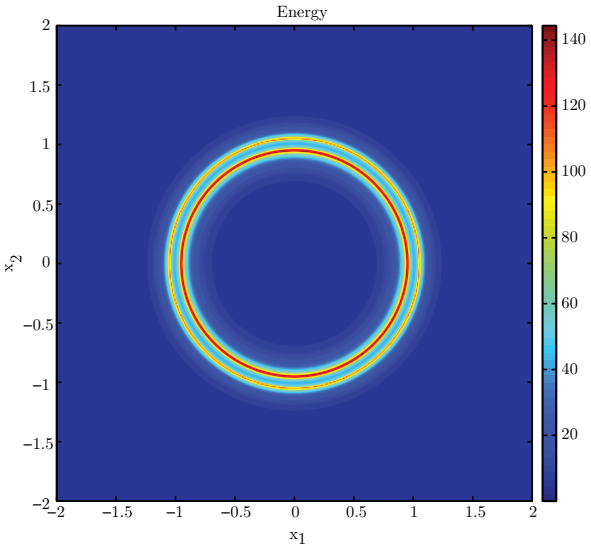

(b)

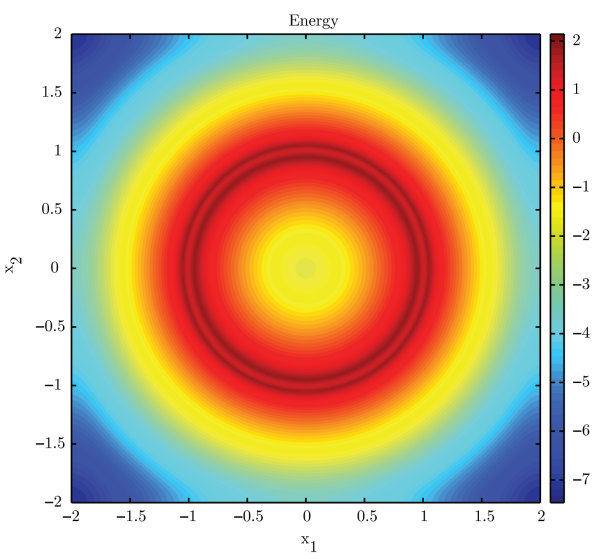

(d)

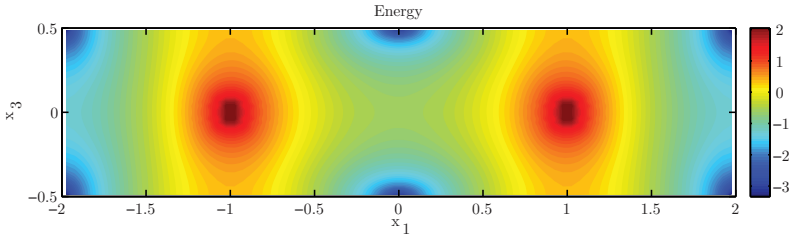

(e)

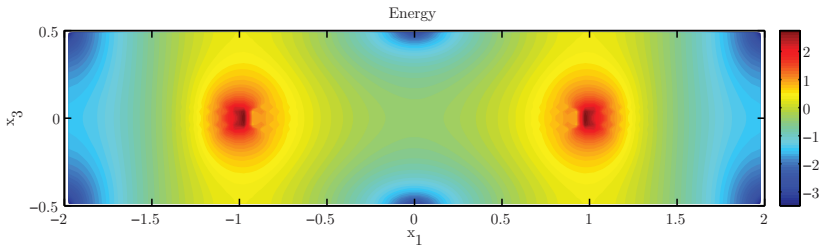

(f)

FIG. 13. Energy plot of the twist disclination for square-shaped and circular loops: (a) and (b) are the contours of the normalized energy density, where $x_{3}=0$; (c) and (d) show the decay of the same contours in a logarithmic scale. Also, (e) and (f) are the logarithmic contour plots of the energy density in $x_{1}-x_{3}$ plane, where $x_{2}=0$. 


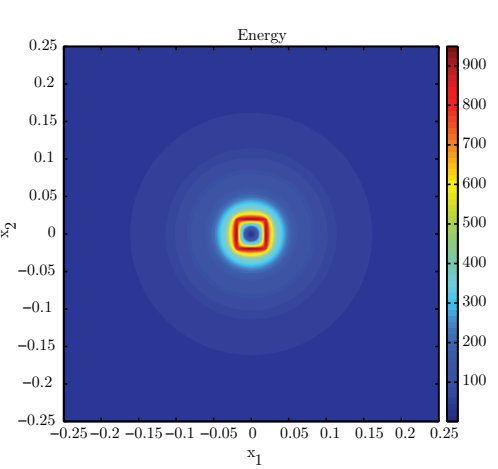

(a)

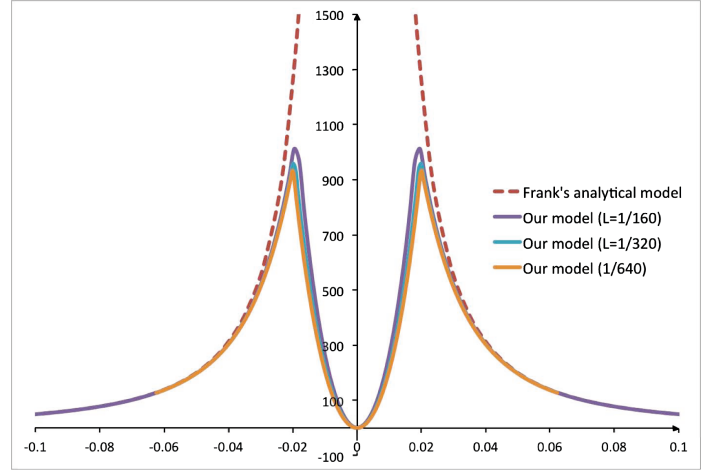

(b)

FIG. 14. (a) Normalized energy density contour of wedge disclination including the defect core and (b) the decay of energy density when $x_{2}=0$ for different element sizes $L$.

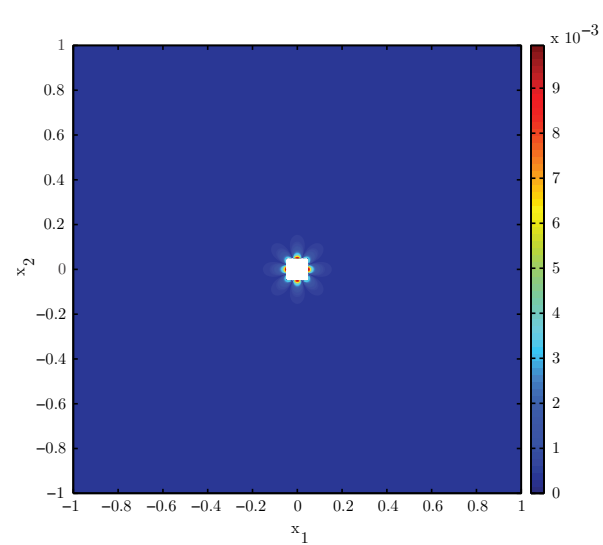

(a)

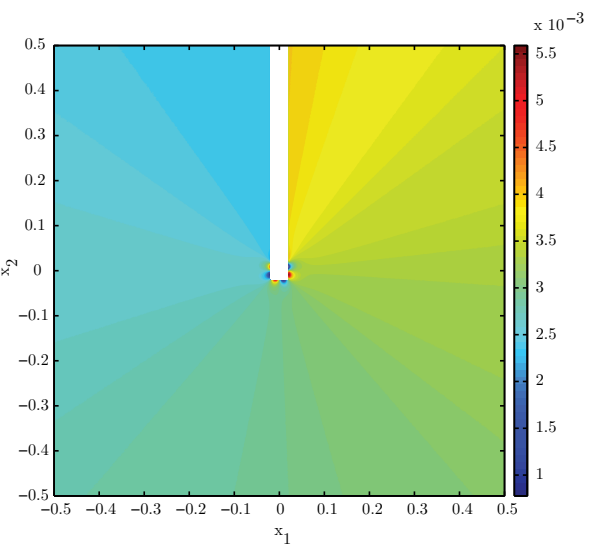

(b)

FIG. 15. Comparison of the computed results from the model with Frank's model: (a) Relative error of energy for wedge disclination. (b) Relative error of director field for wedge disclination. 


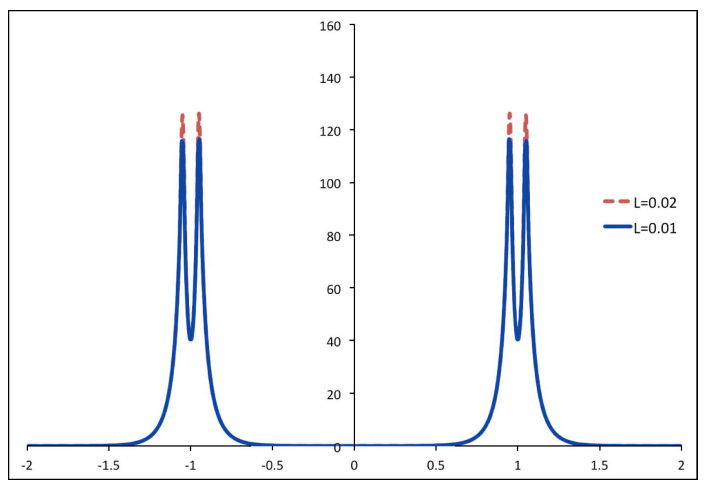

FIG. 16. Normalized energy density profile of the twist disclination for the rectangular loop in Figure $13(\mathrm{a})$ when $x_{2}=0$. The element sizes are $L=0.02$ (red, dashed line) and $L=0.01$ (blue, solid line). 
Acknowledgments. The authors thank the Department of Civil \& Environmental Engineering at Carnegie Mellon University and the NSF (DMS-0811029; PI: N. J. Walkington) for financial support provided to the first author. The writing of this paper was accomplished while the first author was supported on NSF grant CAREER-1150002 awarded to the third author.

\section{REFERENCES}

[Ach01] A. Acharya. A model of crystal plasticity based on the theory of continuously distributed dislocations, Journal of the Mechanics and Physics of Solids 49(4):761-784, 2001.

[Ach10] Amit Acharya, New inroads in an old subject: plasticity, from around the atomic to the macroscopic scale, J. Mech. Phys. Solids 58 (2010), no. 5, 766-778, DOI 10.1016/j.jmps.2010.02.001. MR2642309 (2011e:74025)

[AD12] Amit Acharya and Kaushik Dayal, Continuum mechanics of line defects in liquid crystals and liquid crystal elastomers, Quart. Appl. Math. 72 (2014), no. 1, 33-64, DOI 10.1090/S0033-569X-2013-01322-X. MR.3185131

[AMZ10] Amit Acharya, Karsten Matthies, and Johannes Zimmer, Travelling wave solutions for a quasilinear model of field dislocation mechanics, J. Mech. Phys. Solids 58 (2010), no. 12, 2043-2053, DOI 10.1016/j.jmps.2010.09.008. MR2757690 (2011i:74023)

[AT11] Amit Acharya and Luc Tartar, On an equation from the theory of field dislocation mechanics, Boll. Unione Mat. Ital. (9) 4 (2011), no. 3, 409-444. MR2906769

[BS05] Paolo Biscari and Timothy J. Sluckin, Field-induced motion of nematic disclinations, SIAM J. Appl. Math. 65 (2005), no. 6, 2141-2157 (electronic), DOI 10.1137/040618898. MR2177743 (2006j:76011)

[BS12] Paolo Biscari and Timothy J. Sluckin, A perturbative approach to the backflow dynamics of nematic defects, European J. Appl. Math. 23 (2012), no. 1, 181-200, DOI 10.1017/S0956792510000343. MR2873031

[BZ07] J. M. Ball and A. Zarnescu, Orientable and non-orientable director fields for liquid crystals, PAMM 7(1):1050701-1050704, 2007.

[CAK09] B. G. Chen, G. P. Alexander, and R. D. Kamien, Symmetry breaking in smectics and surface models of their singularities, Proceedings of the National Academy of Sciences 106(37):15577-15582, 2009.

[CF02] Paolo Cermelli and Eliot Fried, The evolution equation for a disclination in a nematic liquid crystal, R. Soc. Lond. Proc. Ser. A Math. Phys. Eng. Sci. 458 (2002), no. 2017, 1-20, DOI 10.1098/rspa.2001.0876. MR1879457 (2002m:76005)

[CK72] P. E. Cladis and M. Kléman, Non-singular disclinations of strength $S=+1$ in nematics, Journal de Physique 33(5-6):591-598, 1972.

[CP89] J. Carr and R. L. Pego, Metastable patterns in solutions of $u_{t}=\epsilon^{2} u_{x x}-f(u)$, Comm. Pure Appl. Math. 42 (1989), no. 5, 523-576, DOI 10.1002/cpa.3160420502. MR997567 (90f:35091)

[DeW73] R. DeWit, Theory of disclinations: IV. Straight disclinations, Journal of Research of the National Bureau of Standards - A. Physics and Chemistry 477A(5):607-658, 1973.

[Eri91] J. L. Ericksen, Liquid crystals with variable degree of orientation, Arch. Rational Mech. Anal. 113 (1990), no. 2, 97-120, DOI 10.1007/BF00380413. MR1079183 (92h:76010)

[Eri95] J. L. Ericksen, Remarks concerning forces on line defects, Theoretical, experimental, and numerical contributions to the mechanics of fluids and solids, Z. Angew. Math. Phys. 46 (1995), no. Special Issue, S247-S271. MR1359323 (97g:73088)

[Esh80] J. D. Eshelby, The force on a disclination in a liquid crystal, Philosophical Magazine A 42(3):359-367, 1980.

[Fra58] F. C. Frank, I. Liquid Crystals. On the Theory of Liquid Crystals, Discuss. Faraday Soc., 25:19-28, 1958.

[Han06] M. Hancock, Method of Green's functions, Lecture notes, 2006.

[HKGLGCC08] D. Harley Klein, L. Gary Leal, C. J. García-Cervera, and H. D. Ceniceros, Threedimensional shear-driven dynamics of polydomain textures and disclination loops in liquid crystalline polymers, Journal of Rheology 52(3):837-863, 2008. 
[HR06] D. K. Hwang and A. D. Rey, Computational studies of optical textures of twist disclination loops in liquid-crystal films by using the finite-difference time-domain method, J. Opt. Soc. Am. A 23(2):483-496, Feb. 2006.

[KB85] Kyozi Kawasaki and Helmut R. Brand, Gauge theory of continuous media with topological defects: uniaxial nematic liquid crystals and superfluid ${ }^{4} \mathrm{He}, \mathrm{Ann}$. Physics 160 (1985), no. 2, 420-440, DOI 10.1016/0003-4916(85)90151-4. MR792172 (86i:82053)

[KF08] M. Kleman and J. Friedel, Disclinations, dislocations, and continuous defects: a reappraisal, Rev. Modern Phys. 80 (2008), no. 1, 61-115, DOI 10.1103/RevModPhys.80.61. MR2399129(2009d:82149)

[KL03] M. Kléman and O. D. Lavrentovich, Soft Matter Physics: An Introduction, Partially Ordered Systems, Springer, 2003.

[Klé73] M. Kléman, Defect densities in directional media, mainly liquid crystals, Philosophical Magazine 27(5):1057-1072, 1973.

[Krö81] E. Kröner, Continuum theory of defects, In: Physics of Defects, Les Houches Summer School, pp. 215-315, 1981.

[Krö93] E. Kröner, Configurational and material forces in the theory of defects in ordered structures, In Materials Science Forum, volume 123, pages 447-454, Trans. Tech. Publ., 1993.

[KVicv99] S. Kralj, E. G. Virga, and S. Žumer, Biaxial Torus Around Nematic Point Defects, Phys. Rev. E, 60:1858-1866, Aug. 1999.

[Les92] F. M. Leslie, Continuum theory for nematic liquid crystals, Contin. Mech. Thermodyn. 4 (1992), no. 3, 167-175, DOI 10.1007/BF01130288. MR1179949 (93f:76004)

[LL00] Fang-Hua Lin and Chun Liu, Existence of solutions for the Ericksen-Leslie system, Arch. Ration. Mech. Anal. 154 (2000), no. 2, 135-156, DOI 10.1007/s002050000102. MR 1784963 (2003a:76014)

[LW00] Chun Liu and Noel J. Walkington, Approximation of liquid crystal flows, SIAM J. Numer. Anal. 37 (2000), no. 3, 725-741 (electronic), DOI 10.1137/S0036142997327282. MR 1740379 (2000k:65174)

[MZ10] Apala Majumdar and Arghir Zarnescu, Landau-De Gennes theory of nematic liquid crystals: the Oseen-Frank limit and beyond, Arch. Ration. Mech. Anal. 196 (2010), no. 1, 227-280, DOI 10.1007/s00205-009-0249-2. MR.2601074 (2011g:82122)

[Sar82] Sarben Sarkar, Hydrodynamics of nematic liquid crystals in the presence of a continuous density of disclinations, Journal of Physics C: Solid State Physics 15(32):6513, 1982.

[Set85] James P. Sethna, Frustration, curvature, and defect lines in metallic glasses and the cholesteric blue phase, Physical Review B 31(10):6278, 1985.

[SKH95] A. Sonnet, A. Kilian, and S. Hess, Alignment tensor versus director: Description of defects in nematic liquid crystals, Phys. Rev. E 52:718-722, Jul 1995.

[SS87] N. Schopohl and T.J. Sluckin, Defect core structure in nematic liquid crystals, Physical Review Letters 59(22):2582-2584, 1987.

[Ste04] I. W. Stewart, The Static and Dynamic Continuum Theory of Liquid Crystals, Taylor and Francis, 2004.

[Wil67] J. R. Willis, Second-order effects of dislocations in anisotropic crystals, International Journal of Engineering Science 5(2):171 - 190, 1967.

[YFMW09] X. Yang, M. Forest, W. Mullins, and Q. Wang, Dynamic defect morphology and hydrodynamics of sheared nematic polymers in two space dimensions, Journal of Rheology 53(3):589-615, 2009. 